\title{
Knots in handlebodies with nontrivial handlebody surgeries
}

\author{
R. Sean Bowman
}

September 23, 2021

\begin{abstract}
We give examples of knots in a genus 2 handlebody which have nontrivial Dehn surgeries yielding handlebodies and show that these knots are not 1-bridge.
\end{abstract}

\section{Introduction}

Let $K$ be a knot in a handlebody $H$ of genus $g$. It is a natural question to ask when $K$ has a nontrivial Dehn surgery yielding a handlebody. When $K$ is isotopic into $\partial H$, there are infinitely many surgeries on $K$ yielding handlebodies homeomorphic to $H$. Berge and Gabai [1, 7] have given examples of handlebodies of genus 2 containing knots which are not isotopic to the boundary yet have nontrivial handlebody surgeries. More recently, Frigerio, Martelli, and Petronio have given infinitely many examples of knots in handlebodies of every genus $g>1$ which have exactly three handlebody surgeries [5].

We say that $K$ is 1 -bridge in $H$ if $K$ is isotopic to $\alpha \cup \beta$, where $\alpha \subseteq \partial H$ is an arc, $\beta$ is properly embedded in $H$, and there is an $\operatorname{arc} \beta^{\prime} \subseteq \partial H$ so that $\beta \cup \beta^{\prime}$ bounds a disk. Berge $[3$ gave examples of 1 -bridge knots in solid tori which are not isotopic to the boundary and have surgeries yielding solid tori. Together with results of Gabai [6], who showed that knots in solid tori with solid tori surgeries must either be isotopic to the boundary or 1-bridge, this leads to a classification of such knots when $g=1$ [2, 7].

The examples of knots in genus $g>1$ handlebodies with handlebody surgeries given in [1, 5, 7] are all 1-bridge knots. Wu [15] conjectured that Gabai's result should hold in this case, that is, if $K$ has a nontrivial surgery yielding a handlebody homeomorphic to $H$, then $K$ is 1 -bridge in $H$. In this paper we give examples of knots in a genus 2 handlebody which disprove this conjecture. The exteriors of these knots are atoroidal, anannular, and have exactly two fillings homeomorphic to handlebodies. 
Specifically, we construct a family of knots $K_{p, q}^{L}$ in a genus 2 handlebody $M_{p, q}^{L}$ parametrized by two integers $p, q$ and a 2 -bridge knot $L$. The handlebody $M_{p, q}^{L}$ contains an embedded separating 3 -punctured sphere $P$ which cuts $M$ into two handlebodies $J$ and $H$, with $K \subseteq H$. We show

Theorem 1.1. The exteriors of the knots $K_{p, q}^{L} \subseteq M_{p, q}^{L}$ are atoroidal, anannular, and have exactly two fillings homeomorphic to handlebodies. Furthermore, $K_{p, q}^{L}$ is not 1-bridge in $M_{p, q}^{L}$ when either

1. $q=p+1$ and $p>1$, or

2. $q=2 p \pm 1, p>1$, and $q>3$.

Proof. The first properties follow from Proposition 4.9 and Proposition 5.7. For the first case, let $(M, H, K)=\left(M_{p, p+1}^{L}, H_{p, p+1}^{L}, K_{p, p+1}^{L}\right)$ and suppose that $K$ is 1bridge in $M$. By Proposition 3.2 there is a tunnel $t$ so that $M^{\prime}=\overline{M \backslash(K \cup t)}$ is a handlebody. Lemma 7.1 shows that we may take $t$ to lie entirely in $H$. According to Lemma 5.4 the surface $P$ is $\partial$-compressible in $M^{\prime}$, and it must $\partial$-compress in the handlebody $H^{\prime}=\overline{H \backslash N(K \cup t)}$ by Lemma 4.1. But by Lemma 9.1, such $\partial$-compressing disks do not exist.

For the second case, the proof proceeds as above except that Lemma 9.3 shows that $P$ is $\partial$-incompressible. This contradicts Lemma 5.4.

The paper is organized as follows: in section 2 we review some basic definitions. Section 3 describes a characterization of 1-bridge knots as those admitting a certain type of tunnel. In section 4, we describe a large family of knots in handlebodies and examine some properties of these knots. The results of section 5 show that these knots admit exactly two Dehn surgeries yielding handlebodies. In section 6 , we prove that infinitely many of the knots are distinct up to homeomorphism of the handlebody. In section 7 we examine the position of tunnels described in section 3 , and in section 8 we prove some technical lemmas about $\partial$-compressing disks in an associated manifold. Finally, in section 9 we show that two subfamilies of the knots described in section 4 are not 1-bridge.

The author would like to thank the referee, Robert Myers, and especially John Luecke for many valuable conversations and suggestions. This work is partially supported by NSF RTG grant DMS-0636643.

\section{Definitions}

Let $S$ be a surface with boundary. An arc $\alpha$ properly embedded in $S$ is called essential if it does not cobound a disk with a subarc of $\partial S$. Otherwise, it is trivial or $\partial$-parallel. A disk $D$ properly embedded in a 3 -manifold $M$ is called essential if $\partial D$ does not bound a disk in $\partial M$. We call an essential disk in a handlebody a meridian disk. 
If $S$ is a surface properly embedded in a 3 -manifold $M$, we say that $S$ is compressible if there is an essential simple closed curve in $S$ which bounds an embedded disk in $M$. Otherwise $S$ is incompressible. An incompressible surface $S$ is $\partial$-compressible if either $S$ is a disk which is isotopic into $\partial M$ or there exists a disk $D$ in $M$ such that $D \cap S=\alpha$ is an essential arc in $S, D \cap \partial M=\beta$ is an arc in $\partial D, \partial D=\alpha \cup \beta$, and $\alpha \cap \beta=\partial \alpha=\partial \beta$. Otherwise, $S$ is $\partial$-incompressible in $M$.

If $S$ is an embedded subsurface of $\partial M$, we say that $S$ is $\partial$-compressible if there is an essential disk $D$ in $M$ such that $\partial D$ intersects $S$ in a single arc essential in $S$. Otherwise $S$ is $\partial$-incompressible.

Let $M$ be an orientable 3-manifold with boundary, and let $D_{0}$, and $D_{1}$ be a pair of disjoint disks in $\partial M$. We say that the manifold $M \cup_{D_{0} \cup D_{1}} D^{2} \times I$ is obtained from $M$ by attaching a 1-handle, where $D^{2} \times \partial I$ is identified with $D_{0} \cup D_{1}$ in such a way as to obtain an orientable manifold. The cocore of the 1-handle $D^{2} \times I$ is $D^{2} \times\{1 / 2\}$.

Let $\alpha$ be a simple closed curve in the boundary of a 3-manifold $M$, and let $A$ be a regular neighborhood of $\alpha$ in $\partial M$. We say that the manifold $M \cup_{A}\left(D^{2} \times I\right)$ is obtained from $M$ by attaching a 2-handle along $\alpha$, where $A$ and $\partial D^{2} \times I$ are identified. We let $M[\alpha]$ denote the manifold obtained by attaching a 2-handle to $M$ along $\alpha$, and call the curve $\alpha$ the attaching curve of the 2 -handle.

We say that a simple closed curve $\gamma$ in the boundary of handlebody $H$ is primitive if attaching a 2 -handle to $H$ along $\gamma$ yields another handlebody. Note that this is equivalent to the existence of a meridian disk for $H$ which meets $\gamma$ in exactly one point.

If $F$ is a submanifold of $M$, denote by $N_{M}(F)$ a closed regular neighborhood of $F$ in $M$. When it is clear from the context what $M$ is, we simply write $N(F)$.

Let $\alpha$ be a simple closed curve in a surface $S$ embedded in a 3-manifold $M$. The isotopy class in $\partial N(\alpha)$ of the curves $\partial N(\alpha) \cap S$ is called the surface slope of $\alpha$ with respect to $F$.

If $K$ is a knot embedded in $S^{3}$, denote $E(K)=\overline{S^{3} \backslash N(K)}$, the exterior of $K$. More generally, if $K$ is a knot embedded in some 3 -manifold $M$, the exterior of $K$ in $M$ is the manifold $\overline{M \backslash N(K)}$.

We adopt the language of [13] for rational tangles. A tangle is a pair $(B, t, m)$ where $B$ is a 3 -ball, $t=t_{1} \cup t_{2}$ is a pair of arcs properly embedded in $B$, and $m$ is a simple closed curve on $\partial B$ which divides $\partial B$ into two disks each containing two points of $\partial t$. We will say that two tangles $(B, t, m)$ and $\left(B, t^{\prime}, m^{\prime}\right)$ are equivalent if there is a homeomorphism of the triples which is the identity on $\partial B$.

A tangle $(B, t, m)$ is rational if $(B, t, m)$ is equivalent to the trivial tangle $\left(D^{2},\{x, y\}\right) \times I$ for $x, y \in D^{2}$. Here $m$ is the vertical circle which bounds a 
disk separating $t_{1}$ and $t_{2}$ in $B$. In this case, $t$ is isotopic rel $\partial t$ to a pair of $\operatorname{arcs}$ on $\partial B$ of slope $r / s \in \mathbb{Q} \cup\{1 / 0\}$.

For a rational number $q$, denote the ceiling of $q$ by $\lceil q\rceil$ and the floor of $q$ by $\lfloor q\rfloor$.

\section{Characterization of 1 -bridge knots}

In this section we give a characterization of 1-bridge knots as those possesing a certain type of tunnel.

Lemma 3.1. Let $\beta \subseteq H$ be an arc properly embedded in a handlebody. Then there is an embedded disk $D$ such that $\partial D=\beta \cup \beta^{\prime}, \beta^{\prime} \subseteq \partial H$, and $\partial \beta=\partial \beta^{\prime}$, iff $\overline{H \backslash N(\beta)}$ is a handlebody.

Proof. Suppose that there is a disk $D$ as in the claim. After cutting $H^{\prime}=$ $\overline{H \backslash N(\beta)}$ along $D \cap H^{\prime}$ we obtain an handlebody homeomorphic to $H$. On the other hand, the space $H$ is obtained from $H^{\prime}$ by adding a 2 -handle. Let $\gamma$ be the attaching curve of the 2 -handle, and note that $\gamma$ is primitive in $H^{\prime}$. Therefore there is a disk $D^{\prime} \subseteq H^{\prime}$ meeting $\alpha$ exactly once. This disk extends to a disk $D \subseteq H$ with $\partial D=\alpha \cup \beta$ and $\alpha \subseteq \partial H$.

If an $\operatorname{arc} \beta$ satisfies these conditions we say that $\beta$ is unknotted.

Let $H$ be a handlebody of genus $g>0$.

Proposition 3.2. A knot $K \subseteq H$ is 1-bridge iff there is an embedded arc $t \subseteq H$ so that $\partial t=\{a, b\}, K \cap t=\{a\}, \partial H \cap t=\{b\}$, and $\overline{H \backslash N(t \cup K)}$ is a handlebody.

Proof. Suppose $K$ is isotopic to $\alpha \cup \beta$, where $\alpha \subseteq \partial H$ and $\beta$ is unknotted. Using a collar neighborhood of $\partial H$, push $\alpha$ and $\beta$ inside $H$ slightly to obtain an isotopic curve $\alpha^{\prime} \cup \beta^{\prime}$. A point $p \in \operatorname{int} \alpha$ traces an arc $t$ under this isotopy. We can use $t$ to push the arc $\alpha^{\prime}$ to the boundary, showing that $\overline{H \backslash N\left(\alpha^{\prime} \cup \beta^{\prime} \cup t\right)} \cong \overline{H \backslash N(\beta)}$. However, $\beta$ is unknotted, so this last space is a handlebody.

On the other hand, if $t$ is an arc as in the proposition, we may slide $K$ along $t$ in a neighborhood of $t$ to the form $\alpha \cup \beta$, where $\alpha \subseteq \partial H$ and $\beta$ is a properly embedded arc in $H$. Then $\overline{H \backslash N(\beta)} \cong \overline{H \backslash N(K \cup t)}$, and we can think of $N(\beta)$ as a 2-handle attached to $\overline{H \backslash N(\beta)}$ along a curve $\gamma$ to obtain $H$. Clearly $\gamma$ is primitive in $\overline{H \backslash N(\beta)}$, so there is a meridian disk $D$ of $\overline{H \backslash N(\beta)}$ whose boundary meets $\gamma$ exactly once. This shows that $\beta$ is unknotted in $H$, and therefore $K$ is 1 -bridge. 


\section{The Knots}

In this section we present a large family of knots in a genus 2 handlebody which have nontrivial handlebody surgeries. The knots are constructed by gluing a genus two handlebody containing a knot to another genus 2 handlebody along a 3-punctured sphere. This 3 -punctured sphere becomes an embedded incompressible surface which is $\partial$-incompressible in the complement of the knot. Furthermore, the knot exteriors contain no essential tori or annuli. We then prove some facts about these knots which will be useful later.

Let $m$ and $l$ be a meridian and longitude, respectively, in the boundary of a solid torus $D^{2} \times S^{1}$, and let $c$ be the core curve of the solid torus. Identify $\overline{D^{2} \times S^{1} \backslash N(c)}$ with $T^{2} \times I$ where $I$ is the interval $[0,1]$ and $T^{2} \times\{1\}=\partial N(c)$. Let $K$ be a $(p, q)$ curve in $T^{2} \times\{0\}$, i.e., a curve meeting $m$ algebraically and geometrically in $p$ points and meeting $l$ algebraically and geometrically in $q$ points. Let $e$ be a curve in $T^{2} \times\{0\}$ parallel to $K$ with $K \cap e=\emptyset$, and let $\pi$ be a curve in $T^{2} \times\{0\}$ meeting $K$ exactly once and $e$ exactly once. The annuli $e \times I$ and $\pi \times I$ meet in a single arc $a$ and restrict to disks in the handlebody $H=\overline{T^{2} \times I \backslash N(a)}$. The disk $D=(\pi \times I) \cap H$ marks $K$ as a primitive curve in $\partial H$, and $E=(e \times I) \cap H$ gives a nonseparating disk which is disjoint from $K$.

Push $K$ to the interior of $H$ and denote by $\left(H_{p, q}, K_{p, q}\right)$ the pair of handlebody and knot obtained by this procedure. We will consider two pairs $(H, K)$ and $\left(H^{\prime}, K^{\prime}\right)$ to be equivalent if there is an orientation preserving homeomorphism $f: H \rightarrow H^{\prime}$ such that $f(K)=K^{\prime}$. If this is the case we write $(H, K) \cong\left(H^{\prime}, K^{\prime}\right)$.

Let $x$ be the boundary component of $m \times I$ lying on $\partial N(c) \cap H$. Then $x$ meets $\partial E$ in $p$ points. Let $s$ be a subarc of $\partial E$ with one endpoint on $x$ and the other on $l$. Let $P$ be a neighborhood of $x \cup s \cup l$ in $\partial H$. This surface is a 3-punctured sphere in $\partial H$ whose complement $R=\overline{\partial H \backslash P}$ is also a 3 -punctured sphere. Call $\partial_{1} P$ the component of $\partial P$ which is homotopic to $x$ in $\partial H, \partial_{2} P$ the component which is homotopic to $l$, and $\partial_{3} P$ the third boundary component. Note that $H$ is homeomorphic to $P \times I$, and in particular, $P$ is incompressible in $H$.

Choose curves $m^{\prime}$ and $l^{\prime}$ in $T^{2} \times\{0\}$ parallel to $m$ and $l$, respectively, so that $D_{m}=\left(m^{\prime} \times I\right) \cap H$ and $D_{l}=\left(l^{\prime} \times I\right) \cap H$ are meridian disks in $H$ meeting $K$ in $p$ and $q$ points. Note that the pair is a complete disk system for $H$, i.e., cutting $H$ along these two disks yields a ball.

Pictured in Figure 1 is $H_{3,4}$ together with $\partial E$ and $P$ (shaded, green). Also shown is the axis of an involution $\tau$ which interchanges $P$ and $R$ and leaves $K$ invariant. Note that there were two choices for the $\operatorname{arc} s$ above. The involution $\tau$ shows that the pair $(H, P)$ is well defined.

There is another involution $\sigma$ on $H$ which interchanges $D_{m}$ and $D_{l}$. Under this involution, shown in Figure 2, $P$ is invariant: $\partial_{3} P$ is sent to itself, and $\partial_{1} P$ and $\partial_{2} P$ are swapped. Note that the attaching curve of the 2-handle $N(a)$ can be taken to be invariant under this involution. The involution shows that 


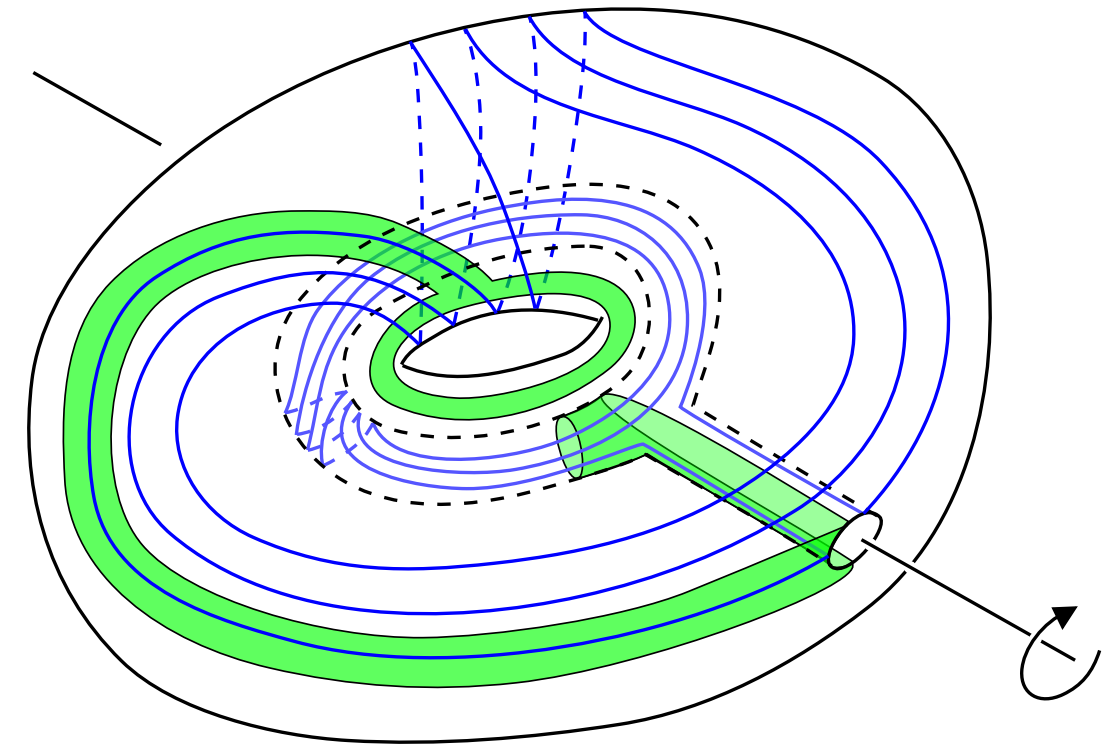

Figure 1: $H_{3,4}$ together with $\partial E$ and $P$

$\left(H_{p, q}, K_{p, q}\right) \cong\left(H_{q, p}, K_{q, p}\right)$

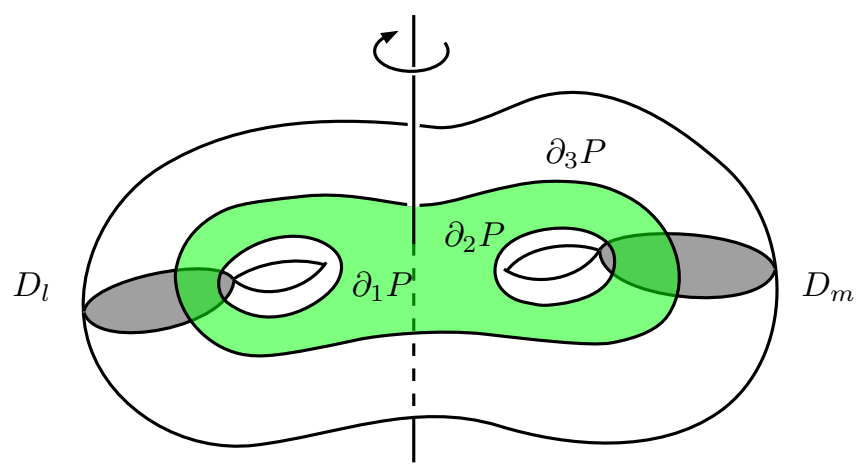

Figure 2: Another view of $H_{p, q}$ with an involution

We obtain the knots we wish to study by gluing $H_{p, q}$ to another handlebody $J$ along $P$. Here we describe the construction of $J$. Let $L$ be a nontrivial 2-bridge knot in $S^{3}$, in 2-bridge position with respect to some height function $h: S^{3} \rightarrow \mathbb{R}$. Then $L$ has an unknotting tunnel $t$ connecting its two maxima [10, that is, a tunnel so that $J=\overline{S^{3} \backslash N(L \cup t)}$ is a handlebody. The knot exterior $E(L)$ is obtained from $J$ by attaching the 2-handle $N(t)$ along an attaching curve $\gamma \subseteq \partial J$. Let $P^{\prime}$ be a 3-punctured sphere embedded in $\partial J$ with $\partial P^{\prime}=\alpha \cup \beta \cup \gamma$, where $\alpha$ and $\beta$ are meridians of $L$. 
If we attach a 2 -handle to $J$ along $\alpha$ or $\beta$, we obtain a new knot exterior whose knot has one maximum and one minimum with respect to $h$. This must be the unknot, and therefore $\alpha$ and $\beta$ are primitive curves. On the other hand, $\gamma$ is not primitive, since attaching a 2 -handle along it gives the exterior of a nontrivial knot. Note that $\alpha$ and $\beta$ are jointly primitive in the sense that $J[\alpha, \beta]$ is a 3 -ball. Therefore we may write them as the cores of the left and right handles of $J$. After embedding $J$ in $S^{3}$ so that both $\alpha$ and $\beta$ bound disks in $S^{3} \backslash H$, we see that there is an $\operatorname{arc} c$ properly embedded in $P$ which connects $\alpha$ and $\beta$. This arc is unique up to isotopy. The sphere $S=\partial J[\alpha, \beta]$ is a genus zero Heegaard surface which divides $S^{3}$ into two balls, $B_{0}$ and $B_{1}$. Assume $J \subseteq B_{0}$ and call the cocores of the attached 2-handles $a$ and $b$. These are unknotted arcs in $B_{0}$.

The arc $c \subseteq \partial J$ extends to an arc in $S$ meeting one endpoint of $a$ and one endpoint of $b$. By abuse of notation we will also call this arc $c$. Note that there is an arc $c^{\prime} \subseteq S \backslash(\partial a \cup \partial b)$ such that $c \cap c^{\prime}=\emptyset$ and $a \cup b \cup c \cup c^{\prime}$ is a knot $K_{0}$ in $S^{3}$. Pushing $c$ and $c^{\prime}$ slightly inside $B_{1}$, we see that $S$ is a bridge surface for $K_{0}$. Furthermore, the exterior of $K_{0}$ in $S^{3}$ is homeomorphic to the space obtained by attaching a 2 -handle to $J$ along $\gamma$. We may therefore regard the pair $(J, P)$ as the exterior of a $m / n$ rational tangle with $P$ as the twice punctured disk on the left half sphere. By viewing the pair $(J, P)$ in this way, we will be able to apply the results of 13 regarding disks in $J$.

Let $J$ and $P^{\prime}$ be a handlebody and pants pair as constructed above, and let $\left(H_{p, q}, K_{p, q}\right)$ be the handlebody and knot pair obtained from a $(p, q)$ curve in the boundary of a solid torus as above. Identify $P$ and $P^{\prime}$ so that $\partial_{1} P$ and $\partial_{2} P$ are identified with $\alpha$ and $\beta$. Note that since $H_{p, q} \cong P \times[0,1]$, the resulting space $M_{p, q}^{L}=J \cup H_{p, q}$ is a handlebody and does not depend on the choice of identification of $P$ and $P^{\prime}$ up to equivalence.

When $J$ is viewed as the exterior of an $m / n$ rational tangle as above, it is easy to see that there is an involution of $J$ which swaps $\alpha$ and $\beta$ and leaves $\gamma$ invariant. Together with the involutions $\tau$ and $\sigma$ considered earlier, we see that the pair $\left(M_{p, q}^{L}, K_{p, q}\right)$ is well defined and that $\left(M_{p, q}^{L}, K_{p, q}\right) \cong\left(M_{q, p}^{L}, K_{q, p}\right)$. Therefore from now on we will always assume that $p<q$. We consider $K$ as a knot in the interior of $M_{p, q}^{L}$ and write $\left(M_{p, q}^{L}, K_{p, q}^{L}\right)$ when we need to emphasize the dependence on $L, p$, or $q$.

In section 6 we will show that there are infinitely many distinct such knots. Later we will show that if $L$ is a nontrivial 2-bridge knot and $n>1$ is an integer, $K_{n, n+1}^{L} \subseteq M_{n, n+1}^{L}$ and $K_{n, 2 n \pm 1}^{L} \subseteq M_{n, 2 n \pm 1}^{L}$ are not 1-bridge knots. In order to do this, we need a few facts about $H$ and $J$.

Fix integers $q>p>1$ and a nontrivial 2-bridge knot $L$, and let $(H, K)=$ $\left(H_{p, q}^{L}, K_{p, q}^{L}\right)$.

Lemma 4.1. The surface $P^{\prime}$ is incompressible and $\partial$-incompressible in $J$.

Proof. Suppose that $\left(J, P^{\prime}\right)$ arises from the exterior of an $m / n$ rational tangle as described above. Let $D$ be a compressing disk or a $\partial$-compressing disk whose 
boundary intersects $\partial P^{\prime}$ minimally. Following [13, we say that $D$ is an $(r, s)$ disk if it meets $\partial_{1} P^{\prime} \cup \partial_{2} P^{\prime}$ in $r$ points and $\partial_{3} P^{\prime}$ in $s$ points.

If $D$ is a compressing disk for $P^{\prime}$, then it is a $(0,0)$ disk and [13, Lemma 2.3] implies that $n=0$. If $D$ is a $\partial$-compressing disk for $P^{\prime}$, then $D$ is either a $(1,1)$ disk, a $(2,0)$ disk, or a $(0,2)$ disk. The same lemma implies that either $n=1$ or $\bar{m}$, the $\bmod n$ inverse of $-m$, is nonzero. Since these are all contradictions to the fact that $L$ is nontrivial, $P^{\prime}$ must be incompressible and $\partial$-incompressible in $J$.

Lemma 4.2. After attaching a 2-handle along $\partial_{i} P, K \subseteq H$ becomes a knot in a solid torus which meets a meridian disk algebraically

- $p$ times if $i=1$,

- $q$ times if $i=2$, and

- $q-p$ times if $i=3$.

Proof. The first two are immediate from the construction. To see the third, consider $H$ embedded in $S^{3}$ as shown in Figure 1. After attaching a 2-handle along $\partial_{3} P$, we obtain a solid torus in $S^{3}$ whose complement is also a solid torus. The linking number of a core of the complementary solid torus with $K$ gives the appropriate algebraic intersection number, which is easily seen to be $q-p$.

Lemma 4.3. Up to isotopy, the disk $E$ is the unique nonseparating essential disk in $\overline{H \backslash N(K)}$.

Proof. Suppose there are two nonisotopic nonseparating essential disks in $H$, $E_{1}$ and $E_{2}$, which do not meet $K$. Among all such pairs, choose the one which minimizes $\left|E_{1} \cap E_{2}\right|$. Let $D$ be an essential disk in $H$ meeting $K$ exactly once and with $\left|D \cap E_{1}\right|$ minimal. An innermost curve/outermost arc argument shows that $D \cap E_{1}=\emptyset$.

After compressing $H$ along $E_{1}$ we obtain a solid torus containing a knot $K$. The disk $D$ shows that the boundary of the resulting solid torus is incompressible in the complement of $K$ and therefore $E_{1} \cap E_{2} \neq \emptyset$.

We may assume that $E_{1} \cap E_{2}$ consists of arcs; let $\alpha$ be one outermost in $E_{1}$ which cuts off a subdisk $\delta$ of $E_{1}$ so that the interior of $\delta$ does not meet $E_{2}$. Surgering $E_{2}$ along $\delta$ we obtain two essential disks, one of which must be nonseparating. Call this disk $E_{2}^{\prime}$. The disk $E_{2}^{\prime}$ is disjoint from $E_{2}$ and has at least one fewer arc of intersection with $E_{1}$. Therefore both pairs $\left(E_{2}, E_{2}^{\prime}\right)$ and $\left(E_{1}, E_{2}^{\prime}\right)$ are parallel, and so $E_{1}$ is parallel to $E_{2}$.

Lemma 4.4. The disk $E$ meets $P$ in $p+q-1$ arcs and cannot be isotoped to reduce this intersection.

Proof. From the description it is clear that we can choose $P$ so that $\partial E \cap P$ consists of a single arc joining $\partial_{1} P$ and $\partial_{2} P, p-1$ parallel arcs joining $\partial_{1} P$ and 
$\partial_{3} P$, and $q-1$ parallel arcs joining $\partial_{2} P$ and $\partial_{3} P$. Similarly, $\partial E \cap R$ consists of a single arc joining $\partial_{1} P$ and $\partial_{2} P, p-1$ parallel arcs joining $\partial_{1} P$ and $\partial_{3} P$, and $q-1$ parallel arcs joining $\partial_{2} P$ and $\partial_{3} P$.

Note that $|\partial E \cap P|=\frac{1}{2}\left(\left|\partial E \cap \partial_{1} P\right|+\left|\partial E \cap \partial_{2} P\right|+\left|\partial E \cap \partial_{3} P\right|\right)$. If we could reduce $|\partial E \cap P|$, we would be able to reduce one of $\left|\partial E \cap \partial_{i} P\right|$. In this case, there would be a bigon of intersection of $\partial E$ and $\partial_{i} P$ on $\partial H$, and an innermost such bigon of intersection would lie in $P$ or $R$, giving a trivial arc. However, each of the arcs above is nonseparating, and so this is the minimal intersection of $P$ and $\partial E$.

The disk $E$ is pictured in Figure 3 together with labels for subarcs of $\partial E$. These labels correspond to arcs in Figure 4, which shows the surfaces $P$ and $R$ along with the arcs $P \cap \partial E$ and $R \cap \partial E$ given by Lemma 4.4. The bold arcs represent a collection of parallel arcs. There are three groups of arcs in $P$ : there is a single arc $a^{P}$ with endpoints on $\partial_{1} P$ and $\partial_{2} P$. There are $p-1$ $\operatorname{arcs} d_{\bar{q}}^{P}, d_{2 \bar{q}}^{P}, \ldots, d_{(p-1) \bar{q}}^{P}$ with endpoints on $\partial_{1} P$ and $\partial_{3} P$. Here $\bar{q}$ is the inverse of $q$ in $\mathbb{Z}_{p}$, and the indices are taken modulo $p$, so that $d_{-1}^{P}=d_{p-1}^{P}$. There are $q-1 \operatorname{arcs} b_{(q-1) \bar{p}}^{P}, b_{(q-2) \bar{p}}^{P}, \ldots, b_{\bar{p}}^{P}$ with endpoints on $\partial_{2} P$ and $\partial_{3} P$. Here $\bar{p}$ is the inverse of $p$ in $\mathbb{Z}_{q}$, and the indices are taken modulo $q$ so that $b_{-1}^{P}=b_{q-1}^{P}$.

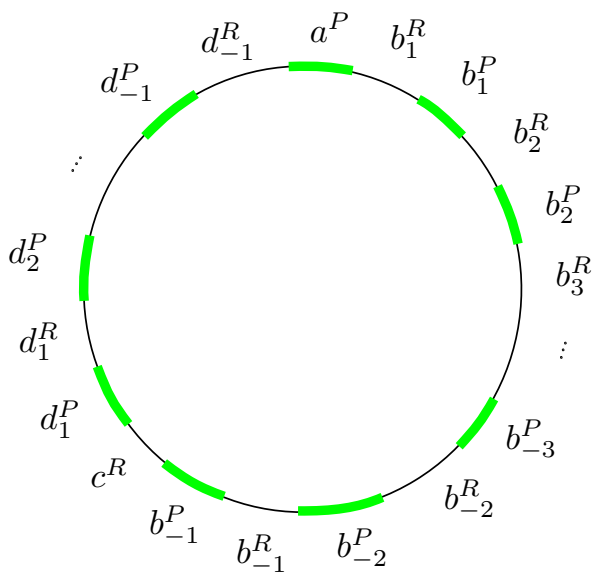

Figure 3: The disk $E$

In $R$, there is a similar collection of arcs. The arc in $R$ with endpoints on $\partial_{1} P$ and $\partial_{2} P$ is called $c^{R}$, but otherwise the names are similar. We will call components of $P \backslash \partial E$ and $R \backslash \partial E$ regions of $P$ and $R$, respectively. All regions of $P$ and $R$ are rectangles except for two hexagons, $H_{1}^{P}$ and $H_{2}^{P}$, in $P$ and two, $H_{1}^{R}$ and $H_{2}^{R}$, in $R$. The boundary of $H_{1}^{P}$ contains the $\operatorname{arcs} d_{\bar{q}}^{P}$ and $b_{\bar{p}}^{P}$, and the boundary of $H_{1}^{R}$ contains $d_{\bar{q}}^{R}$ and $b_{\bar{p}}^{R}$. Similarly, the boundary of $H_{2}^{P}$ contains the $\operatorname{arcs} d_{(p-1) \bar{q}}^{P}$ and $b_{(q-1) \bar{p}}^{P}$, and the boundary of $H_{2}^{R}$ contains $d_{(p-1) \bar{q}}^{R}$ and $b_{(q-1) \bar{p}}^{R}$. When there is no danger of confusion we will drop the superscripts. 


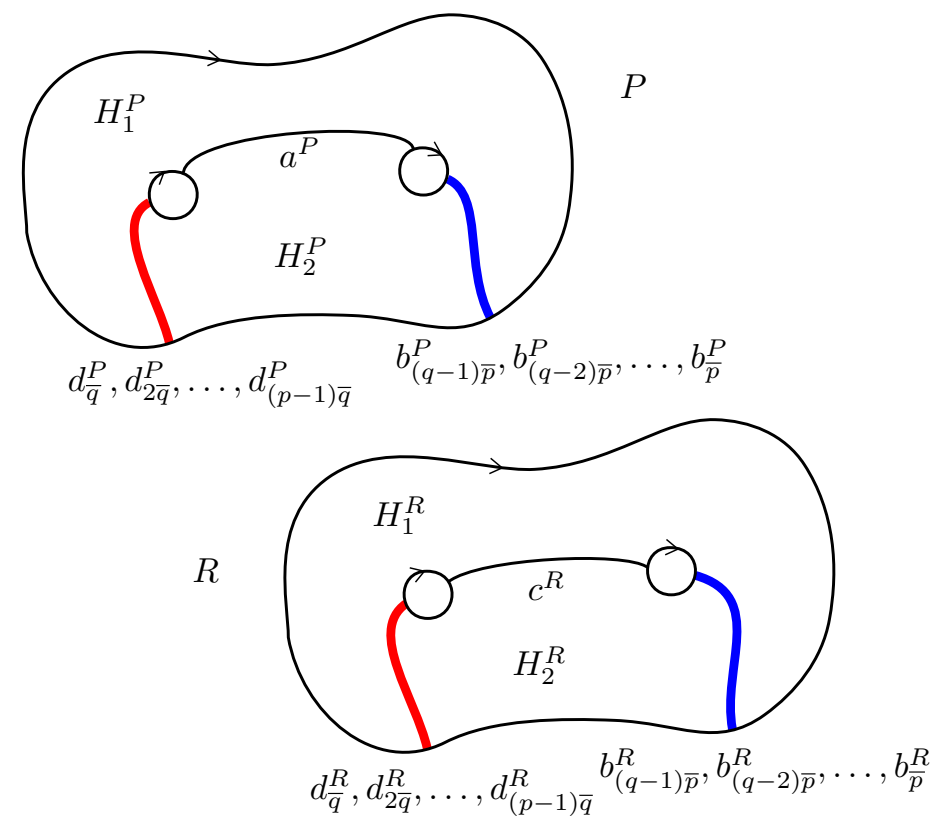

Figure 4: The surfaces $P$ and $R$, and the boundary of $E$

Remark 4.5. There are several things to note about these two figures:

- $a^{P} \cap \partial_{2} P=b_{1}^{R} \cap \partial_{2} P$ and $a^{P} \cap \partial_{1} P=d_{-1}^{R} \cap \partial_{1} P$,

- $c^{R} \cap \partial_{1} P=d_{1}^{P} \cap \partial_{1} P$ and $c^{R} \cap \partial_{2} P=b_{-1}^{P} \cap \partial_{2} P$,

- $b_{i}^{R} \cap \partial_{3} P=b_{i}^{P} \cap \partial_{3} P$,

- $d_{i}^{R} \cap \partial_{3} P=d_{i}^{P} \cap \partial_{3} P$,

- $b_{i}^{P} \cap \partial_{2} P=b_{i+1}^{R} \cap \partial_{2} P$ for $i=1, \ldots, q-2$,

- $d_{i}^{R} \cap \partial_{1} P=d_{i+1}^{P} \cap \partial_{1} P$ for $i=1, \ldots, p-2$, and

- $H_{1}^{P} \cap \partial_{3} P=H_{1}^{R} \cap \partial_{3} P$ and $H_{2}^{P} \cap \partial_{3} P=H_{2}^{R} \cap \partial_{3} P$.

Finally, here are some facts about $H, J, K$, and $M$ which will be used later.

Lemma 4.6. Let $N$ be $H$ or $J$. Any incompressible annulus A properly embedded in $N$ with $\partial A \subseteq P$ may be isotoped to lie entirely in $P$.

Proof. Let $A$ be such an annulus. No two components of $\partial P$ are homologous in $H$ since it is a product. Furthermore, after attaching a 2-handle to $J$ along a component of $\partial P$, the other two components of $\partial P$ become meridians in a knot exterior. Therefore no two components of $\partial P$ are homologous in $N$, and so $\partial A$ bounds an annulus $B \subseteq P$. The surface $A \cup B$ is a torus $T$. Isotop $T$ slightly inside $N$ to obtain an embedded torus. There is an annulus $C$ so that $\partial_{1} C$ lies 
on $P$ and $\partial_{2} C$ is a core of $B$. Since a core of $C$ is isotopic to a component of $\partial P$ in $N$ and $P$ is incompressible in $N, C$ must be incompressible in $N$.

A torus in a handlebody must compress to one side. If $T$ were compressible to the side containing $C$, then using an innermost disk/outermost arc argument we could find a compressing disk $D$ for $T$ not meeting $A$. Therefore we could isotop $D$ so that $\partial D \subseteq A$. But $A$ is incompressible, and so $T$ must be compressible to the side not containing $C$. We conclude that $T$ bounds a solid torus $S$ on this side.

If the core of $B$ were not longitudinal in $S$, we would obtain a reducible manifold after attaching a 2-handle to $N$ along this curve. Examining $J$ and $H$ it is clear that this does not happen, and therefore we can isotop $A$ to $B$ through $S$.

Lemma 4.7. Every $\partial$-compressing disk for $P$ in $H \cong P \times I$ is isotopic to one of the six disks of the form $\lambda \times I$ for an essential arc $\lambda \subseteq P$.

Proof. Suppose we have two $\partial$-compressing disks $D_{1}$ and $D_{2}$ which are not isotopic but which meet $P$ in arcs of the same isotopy class. Among all such disks, choose $D_{1}$ and $D_{2}$ to minimize $\left|D_{1} \cap D_{2}\right|$. If $D_{1} \cap D_{2}=\emptyset$, then after cutting $H$ along $D_{1}$ we can isotop $\partial D_{2}$ to lie in $\partial H \backslash P$. This surface is incompressible, so $D_{2}$ must be trivial here. This means that $D_{2}$ is parallel to $D_{1}$ in $H$.

So assume that $D_{1} \cap D_{2} \neq \emptyset$. Since $\partial D_{1} \cap P$ and $\partial D_{2} \cap P$ are of the same class in $P$, we may isotop $D_{1}$ and $D_{2}$ so that $\partial D_{1} \cap \partial D_{2} \cap P$ is empty. This isotopy does not introduce new intersections, so consider an arc $\alpha$ of $D_{1} \cap D_{2}$ which is outermost in $D_{1}$. This arc cuts off a subdisk $\delta$ of $D_{1}$ whose interior does not meet $D_{2}$. We may surger $D_{2}$ along $\delta$ to obtain a new $\partial$-compressing disk $D_{2}^{\prime}$ with $D_{2} \cap P=D_{2}^{\prime} \cap P$. This disk is disjoint from $D_{2}$ and has at least one fewer arc of intersection with $D_{1}$, and so both pairs $\left(D_{2}, D_{2}^{\prime}\right)$ and $\left(D_{1}, D_{2}^{\prime}\right)$ are parallel. This means that $D_{1}$ and $D_{2}$ are also parallel.

Lemma 4.8. Every $\partial$-compressing disk for $P$ in $H$ meets $K$ at least once.

Proof. Let $D$ be an essential disk in $H$ which does not meet $K$. If $D$ is a $\partial$-compressing disk for $P$, then by Lemma 4.3 and Lemma 4.4 it must be separating. Suppose that $\partial D$ meets $\partial_{i} P$. After compressing along $D$ we obtain two solid tori $S_{1}$ and $S_{2}$. Let $D_{j}$ be the nonseparating $\partial$-compressing disk for $P$ which does not meet $\partial_{j} P$. This disk is unique up to isotopy by the previous lemma. By Lemma 4.2 each of these disks meets $K$ at least once. But $D_{x}$ and $D_{y}$ are meridian disks for $S_{1}$ and $S_{2}$, where $\{i, x, y\}=\{1,2,3\}$. This is impossible since $K$ is contained in either $S_{1}$ or $S_{2}$.

Proposition 4.9. Both the spaces $\overline{H \backslash N(K)}$ and $\overline{M \backslash N(K)}$ are irreducible and atoroidal. The boundary of $N(K)$ is incompressible in $\overline{H \backslash N(K)}$ and $\overline{M \backslash N(K)}$. There are no essential annuli in $\overline{M \backslash N(K)}$.

Proof. Let $F$ be a sphere in $H$ which does not bound a ball in the complement of $K$. Then either $F$ does not bound a ball in $H$ (impossible since handlebodies 
are irreducible) or $F$ bounds a ball containing $K$. However, there are meridian disks in $H$ which have nonzero algebraic intersection with $K$.

Suppose that $T \subseteq \overline{M \backslash N(K)}$ is an essential torus with $|T \cap P|$ minimal. If $|T \cap P|>0$, examine the intersections $T \cap P$. By the minimality of $|T \cap P|$ and incompressibility of $T$ and $P$, we may assume that there are no simple closed curves of intersection of $T \cap P$ which are trivial in $T$ or $P$. So suppose that there is a curve of intersection of $T \cap P$ which is essential in both $P$ and $T$. Since $P$ is separating and $T$ contains no trivial curves of intersection, we can find an annulus $A \subseteq T$ such that int $A$ has no intersections with $P$. By Lemma 4.6, we may isotop $T$ to reduce $|T \cap P|$. This contradiction shows that $T \cap P=\emptyset$ and therefore $T$ lies in $H$. However, this is impossible since $K$ is primitive in $H$.

If $\partial N(K)$ is compressible in $\overline{H \backslash N(K)}$, we see that either $K$ is contained in a ball or $H$ is reducible after compressing. The same holds for $\overline{M \backslash N(K)}$. This contradicts the irreducibility of these spaces.

Finally, suppose that $A$ is an essential annulus in $\overline{M \backslash N(K)}$ chosen to minimize $|A \cap P|$. Using an innermost curve/outermost arc argument and Lemma 4.6 we can show that $A \cap P=\emptyset$. Suppose then that $A$ has one boundary component, $\partial_{1} A$, in $R$ and the other, $\partial_{2} A$, in $\partial N(K)$. Since $K$ does not bound a disk in $H$, The component $\partial_{2} A$ cannot be meridional on $\partial N(K)$ because $A$ would become a compressing disk for $R$ under the trivial surgery. Therefore $\partial_{2} A$ meets the meridian of $\partial N(K)$ at least once, and $\partial_{1} A$ is isotopic to a component $\gamma$ of $\partial P$.

If $\partial_{2} A$ meets a meridian of $\partial N(K)$ more than once, then $\gamma$ is conjugate to an $n$-th power of $K$ in $\pi_{1}(H)$ for $n>1$. Since $\gamma$ and $K$ are both primitive in $\pi_{1}(H)$, this is impossible. Therefore $\partial_{2} A$ is longitudinal on $\partial N(K)$, and $A$ describes an isotopy of $K$ to $\gamma \subseteq R$. This is impossible by Lemma 4.8 .

If $A$ has both components on $\partial M$, then $A$ lies in $J$ or $H$ disjoint from $P$. But then $A$ is either an essential annulus in a hyperbolic knot exterior or an essential annulus in a compression body which is not vertical. Both are impossible.

Lemma 4.10. The boundary of every essential disk in $\overline{H \backslash N(K)}$ meets $P$ in at least three arcs.

Proof. Let $D$ be a meridian disk for $H$ which does not meet $K$. By Lemma 4.8 and the incompressibility of $P$ in $H, D$ meets $P$ in at least two arcs. If $D$ meets $P$ fewer than four times, it must be separating by Lemma 4.3 and Lemma 4.4. So let $D$ be a separating meridian disk for $H$ missing $K$ and meeting $P$ in two essential $\operatorname{arcs} \lambda_{1}$ and $\lambda_{2}$. The disk $D$ cuts $H$ into two solid tori $T$ and $T^{\prime}$ with $K \subseteq T$.

There are two arcs $\kappa_{1}$ and $\kappa_{2}$ parallel to $\lambda_{1}$ so that each component of $P \backslash\left(\kappa_{1} \cup \kappa_{2}\right)$ contains exactly one of $\lambda_{1}$ or $\lambda_{2}$. Let $F_{1}$ and $F_{2}$ denote the product disks $\kappa_{1} \times I, \kappa_{2} \times I \subseteq P \times I$, and choose $D$ to minimize $\left|D \cap\left(F_{1} \cup F_{2}\right)\right|$ with the constraint that $\partial D \cap P$ consists of the $\operatorname{arcs} \lambda_{1}$ and $\lambda_{2}$. Isotop $K$ to minimize 
$\left|K \cap\left(F_{1} \cup F_{2}\right)\right|$ while keeping $K \cap D=\emptyset$. Since $F_{1} \cup F_{2}$ separates $H, D \cap F_{i}$ is nonempty for at least one $i=1,2$. Without loss of generality assume $D \cap F_{1} \neq \emptyset$.

We may assume that there are no simple closed curves of intersection in $D \cap F_{1}$. Let $\alpha$ be an arc of $D \cap F_{1}$, outermost in $F_{1}$, cutting off a subdisk $F_{1}^{\prime}$ of $F_{1}$ whose interior does not meet $D$. If $\alpha$ cuts off a subdisk $\delta$ of $D$ which does not meet $P$, we could surger $D$ along $\delta$ to obtain a new disk meeting $P$ in the $\operatorname{arcs} \lambda_{1}$ and $\lambda_{2}$ and having fewer intersections with $F_{1}$ and $F_{2}$. Therefore $\alpha$ divides $D$ into two halves each containing one of $\lambda_{1}$ or $\lambda_{2}$. Surgering along $F_{1}^{\prime}$ we obtain two parallel $\partial$-compressing disks for $P$ in $H$, and so we have shown that $D$ consists of $D_{1}$ and $D_{2}$, isotopic to product disks, tubed along an $\operatorname{arc}$ in $\partial H \backslash P$.

Reversing the surgery along $F_{1}^{\prime}$ corresponds to adding a 1-handle to one of the components of $H$ cut along $D_{1} \cup D_{2}$, and therefore $F_{1}^{\prime}$ is nonseparating in its solid torus. We claim that $F_{1}^{\prime}$ is a meridian disk for $T$, the solid torus containing $K$. If not, $F_{1}^{\prime} \cap K=\emptyset$, and so $D_{1}$ and $D_{2}$ are $\partial$-compressing disks for $P$ which do not meet $K$, impossible by Lemma 4.8. The exterior of $K$ in $H$ is irreducible and atoroidal by Proposition 4.9, so $K$ is isotopic to a core of $T$ and therefore isotopic into $\partial T \cap(\partial H \backslash P)$. However, $K$ is clearly not isotopic in $H$ to a boundary component of $\partial H \backslash P$, and so no such disks exist.

\section{Surgery on the knots}

Fix a nontrivial 2-bridge knot $L$ and two relatively prime positive integers $p<q$, and let $(K, M)=\left(K_{p, q}^{L}, M_{p, q}^{L}\right)$. In this section we show that the knot $K \subseteq M$ has exactly one nontrivial surgery yielding a handlebody.

Proposition 5.1. The knot $K \subseteq M$ has a nontrivial handlebody surgery.

Proof. We will first show that $K \subseteq H$ has a handlebody surgery under which $P$ becomes $\partial$-compressible. To see this, push $K$ inside $H$ so that it lies in the punctured torus $\left(T^{2} \times\{1 / 2\}\right) \cap H$. Let $E(K)$ be the exterior of $K$ in $H$, and let $S$ be the 3 -punctured sphere $\left(T^{2} \times\{1 / 2\}\right) \cap E(K)$. This surface defines a slope on $\partial N(K)$. Since $K$ is isotopic to a primitive curve on $\partial H$, surgery at this slope yields a handlebody. After surgery, $S$ becomes a disk meeting $P$ in a single essential separating arc.

Perform the $\partial$-compression and glue the resulting two solid tori to $J$. The cores of the gluing annuli are both primitive in $J$ by construction, so the result is a handlebody. Reversing the $\partial$-compression does not change this.

Let $\mu$ be the meridional slope on $\partial N(K)$, and let $\lambda$ be the slope on $\partial N(K)$ given by Proposition 5.1. Let $H(\alpha)$ denote the space resulting from Dehn surgery on $K$ in $H$ along the $\alpha$ slope. Since $K$ is isotopic to a primitive curve in $\partial H, H(\alpha)$ is a handlebody for every slope $\alpha$. 
Proposition 5.2. After attaching a 2-handle to $H(\lambda)$ along $\partial_{3} P$, we obtain a Seifert fiber space over the disk with two exceptional fibers of order $p$ and $q$.

Proof. Recall from Proposition 5.1 that there is a separating $\partial$-compressing disk $D$ for $P$ in $H(\lambda)$. This disk divides $H(\lambda)$ into two solid tori: $T_{1}$ contains $\partial_{1} P$ and $T_{2}$ contains $\partial_{2} P$. Attaching a 2-handle to $H$ along $\partial_{1} P$, we obtain a knot in a solid torus isotopic to a $(p, q)$ curve with respect to $m$ and $l$. The surgery slope $\lambda$ is the surface slope with respect to this embedding, and so surgery yields the connect sum of a solid torus and a lens space of order $p$. Similarly, attaching a 2-handle to $H(\lambda)$ along $\partial_{2} P$ yields the connect sum of a solid torus and a lens space of order $q$.

Let $D_{1}$ and $D_{2}$ be meridian disks for $T_{1}$ and $T_{2}$ disjoint from $D$. Clearly $\mid \partial_{1} P$. $\partial D_{1} \mid=p$ and $\left|\partial_{2} P \cdot \partial D_{2}\right|=q$, and so $H(\lambda)$ appears as in Figure 5. In this figure, $\partial_{1} P$ runs $p$ times around the left handle and $\partial_{2} P$ runs $q$ times around the right handle. With respect to the basis for $\pi_{1}(H(\lambda))$ dual to $\left\{D_{1}, D_{2}\right\}$, the fundamental group of $H(\lambda)\left[\partial_{3} P\right]$ is $\left\langle x, y: x^{p}=y^{q}\right\rangle$. This space is a Seifert fiber space over the disk with exceptional fibers of order $p$ and $q[12$.

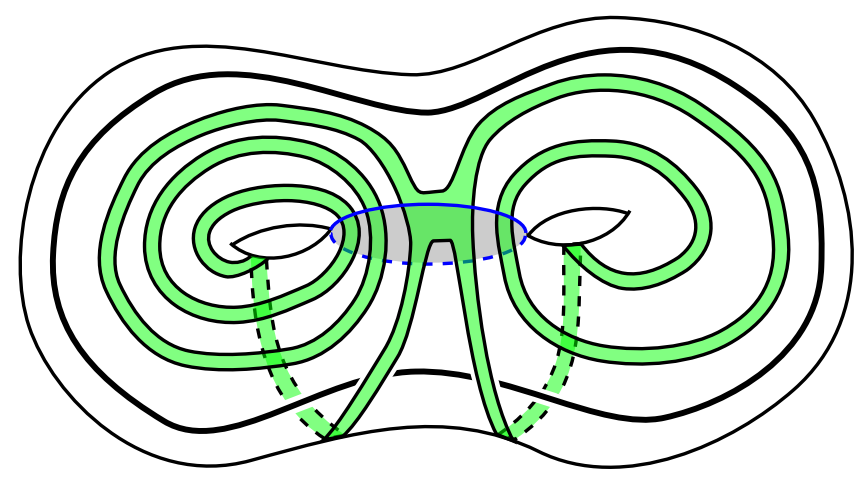

Figure 5: $H(\lambda), K^{\prime}, P$, and $E$

After surgery on $K$, the core of the attached solid torus becomes a new knot in $H(\lambda)$ which we will call the dual knot. Let $K^{\prime} \subseteq H(\lambda)$ be the dual knot to $K$. In light of Proposition 5.2, we get a picture of $H(\lambda)$ as in Figure 5. Notice in particular the disk $E$ and the intersections of its boundary with $P$.

Corollary 5.3. The knot $K$ is not isotopic to the boundary of the solid torus $H\left[\partial_{3} P\right]$.

Proof. Let $N=H\left[\partial_{3} P\right]$ and suppose that $K$ is isotopic to $\partial N$. Recall that $K$ meets a meridian disk for $N$ algebraically in $q-p$ points by Lemma 4.2. Therefore $N(\lambda)$ is either a Seifert fiber space over the disk with two exceptional fibers of order $q-p$ and $m$ for some nonzero integer $m$ or (if $\lambda$ is the surface slope of $K$ in $\partial N)$ a connect sum of a solid torus and lens space. The previous 
result shows that $N(\lambda)$ is a Seifert fiber space over the disk with exceptional fibers of orders $p$ and $q$. This space is irreducible, so it cannot be a connect sum. Furthermore, the classification of Seifert fiber spaces (see for example [9]) then shows that $q-p=p$ or $q-p=q$, both of which are impossible.

The following lemma is well known and can be proven using an innermost curve/outermost arc argument.

Lemma 5.4. Let $S \subseteq M$ be a properly embedded incompressible surface in a handlebody of genus $g>0$. Then $S$ is $\partial$-compressible.

The next proposition plays an important role in our argument that many of the knots constructed above are not 1-bridge. In this section we will use it to consider the effect of surgery on $K$ at slopes other than $\mu$ and $\lambda$.

Proposition 5.5. Let $P \subseteq M$ be a properly embedded, separating, incompressible 3-punctured sphere in a handlebody of genus $g>1$, so that $\partial$-compressing $P$ in $M$ yields a separating surface $\mathcal{A}$ consisting of one or two annuli. Then the core (s) of $\mathcal{A}$ are primitive on at least one side of $P$.

Proof. Suppose that $P$ separates $M$ into $M_{1}$ and $M_{2}$. These are easily seen to be handlebodies themselves. Suppose further that $D$ is a $\partial$-compressing disk for $P$ which is properly embedded in $M_{2}$. Let $\mathcal{A}$ be the annulus or annuli resulting from $\partial$-compressing. Then $\mathcal{A}$ separates $M$ into $N_{1}$ and $N_{2}$ where $N_{1} \subseteq M_{1}$ is homeomorphic to $M_{1}$ and $N_{2}$ is either a solid torus or a pair of solid tori. In either case, $M_{2}$ is obtained from $N_{2}$ by attaching a 1-handle.

Let $A$ be a component of $\mathcal{A}$. Clearly $A$ is a properly embedded, incompressible annulus in $M$, and therefore it $\partial$-compresses by Lemma 5.4. Let $D^{\prime}$ be a $\partial$-compressing disk for $A$ in $M$. Using an innermost curve/outermost arc argument, we may assume that $D^{\prime}$ lies entirely in $N_{1}$ or $N_{2}$. If $D^{\prime} \subseteq N_{1}$, then $D^{\prime}$ shows that the core of $A$ is primitive in $M_{1}$ since $M_{1} \cong N_{1}$. If $D^{\prime} \subseteq N_{2}$, then the core of $A$ is primitive in $M_{2}$ since we may choose the attaching disk(s) of the 1-handle to be disjoint from a disk showing that the core of $A$ is primitive.

Lemma 5.6. Let $P$ be a 3-punctured sphere embedded in the boundary of a genus 2 handlebody $H$ so that no two components of $\partial P$ are homologous in $H$ and at least two components of $\partial P$ are primitive in $H$. If $P$ is $\partial$-compressible, then either

- $H \cong P \times I$, or

- there is one component $z$ of $\partial P$ which is not primitive, and every $\partial-$ compressing disk for $P$ is disjoint from $z$.

Proof. First note that if $D$ is nonseparating in $H$ but $\partial D$ separates $P$, two components of $\partial P$ are homologous in the solid torus resulting from $\partial$-compressing. These components must have been homologous in $H$, and therefore $D$ meets two different components of $\partial P$. 
Suppose that there is a component $z$ of $\partial P$ which is not primitive in $H$ and let $D$ be $\partial$-compressing disk for $P$ in $H$. In this case $D$ must be disjoint from $z$ since otherwise it would mark $z$ as primitive.

Therefore suppose that $D$ is separating in $H$ and meets $z$. Cutting $H$ along $D$ we obtain two solid tori each with an annulus embedded in its boundary. The primitive components $x$ and $y$ of $\partial P$ are disjoint from $D$, and so an innermost curve/outermost arc argument shows that they remain primitive in their respective solid tori. Therefore these solid tori are products of the annuli in their boundaries. Reversing the $\partial$-compression preserves the product structure, so $H \cong P \times I$.

The same argument shows that if every component of $\partial P$ is primitive and $D$ is a separating $\partial$-compressing disk for $P$ in $H$, then $H \cong P \times I$.

If $D$ is nonseparating, a similar argument shows that we obtain a single solid torus with an annulus in its boundary whose core is primitive. Again, we see that $H \cong P \times I$.

Proposition 5.7. If surgery on $K$ in $M$ along the slope $\alpha$ yields a handlebody, then $\alpha=\mu$ or $\alpha=\lambda$.

Proof. Let $\alpha \neq \mu, \lambda$ be a slope on $\partial N(K)$ such that surgery on $K$ in $M$ along $\alpha$ yields a handlebody. By Proposition 4.9 and a result of Wu [14, $\Delta(\alpha, \mu)=$ $\Delta(\alpha, \lambda)=1$. Recall that $\partial H$ contains two punctured tori $T_{1}=\left(T^{2} \times\{0\}\right) \cap H$ and $T_{2}=\left(T^{2} \times\{1\}\right) \cap H$. Isotop $K$ to lie in $T_{1}$; the surface slope of $K$ with respect to $\partial H$ is $\lambda$. Note that $K$ is disjoint from $\partial_{2} P$. The effect of $\alpha$ surgery on $\partial H$ can be seen by Dehn twisting along $K$. Since $K$ is disjoint from $\partial_{2} P$, this curve remains primitive in $H(\alpha)$. Similarly, we may isotop $K$ to lie in $T_{2}$ to see that $\partial_{1} P$ remains primitive in $H(\alpha)$.

By a result of $\mathrm{Ni}$ [11], if $H(\alpha) \cong P \times I$ then $K$ meets every nonseparating $\partial$-compressing disk for $P$ in $H$ at most twice. Since this is clearly not true, Lemma 5.6 implies that $\partial_{3} P$ is not primitive in $H(\alpha)$ and that every $\partial$-compressing disk for $P$ in $H(\alpha)$ is disjoint from $\partial_{3} P$.

By Proposition 5.5, $P$ must $\partial$-compress in $M(\alpha)$, and the core(s) of the resulting annulus or annuli must be primitive on at least one side of $P$. Since $\partial_{3} P$ is not primitive in either $J$ or $H(\alpha)$, a $\partial$-compressing disk $D \subseteq H(\alpha)$ for $P$ must meet $\partial_{3} P$. This contradicts Lemma 5.6 and shows that $M(\alpha)$ is not a handlebody.

\section{$6 \quad$ Infinitely many distinct examples}

Here we show that infinitely many of the knots described above are inequivalent in the sense that there is no homeomorphism of the handlebody which carries the first knot to the second. We need a technical lemma which says roughly 
that the isotopy class of the multicurve $\partial P$ is determined by the construction in section 4 :

Lemma 6.1. Fix a hyperbolic 2-bridge knot $L$ and integers $1<p<q$ with $\operatorname{gcd}(p, q)=1$. Suppose that $(M, K)$ and $\left(M^{\prime}, K^{\prime}\right)$ arise from the construction in section 4, and let $P$ and $P^{\prime}$ be the corresponding 3-punctured spheres described in that section. If $\left(M^{\prime}, K^{\prime}\right)$ is homeomorphic to $(M, K)$ by a homeomorphism $h$, then $h\left(\partial P^{\prime}\right)$ is isotopic to $\partial P$.

Proof. We will work in $M$ and identify $P^{\prime}$ with its image under $h$. Note that the roles of $P$ and $P^{\prime}$ are symmetric in the proof.

Isotop $P^{\prime}$ so that $\left|P \cap P^{\prime}\right|$ is minimal. The curves $\partial P$ cut $\partial M$ into two 3punctured spheres, so if $P \cap P^{\prime}=\emptyset$, then $\partial P^{\prime}$ is isotopic to $\partial P$.

If $P \cap P^{\prime} \neq \emptyset$, there are several cases:

1. Suppose that $P \cap P^{\prime}$ contains a simple closed curve which is trivial in $P^{\prime}$. Then it must be trivial in $P$ because both surfaces are incompressible, and so we can isotop to reduce $\left|P \cap P^{\prime}\right|$.

If $P \cap P^{\prime}$ contains an arc which is trivial in $P^{\prime}$, then an outermost such arc gives either a $\partial$-compressing disk for $P$ in the complement of $K$ (impossible by Lemma 4.1 and Lemma 4.8) or a disk which guides an isotopy of $P^{\prime}$ reducing $\left|P \cap P^{\prime}\right|$.

2. If there is a simple closed curve of intersection of $P \cap P^{\prime}$ which is essential in $P^{\prime}$ then it must also be essential in $P$. In this case there is an incompressible annulus $A \subseteq N, N=J$ or $H$, with one boundary component, $\partial_{1} A$, on $P$, and the other, $\partial_{2} A$, on $\partial N \backslash P$. We may isotop $\partial_{2} A$ to lie on $P$ and use Lemma 4.6 to reduce $\left|P \cap P^{\prime}\right|$.

3. If $P \cap P^{\prime}$ consists of a single arc which separates $P^{\prime}$, then there is a separating, incompressible annulus $A$ properly embedded in $H$ with one boundary component, $\partial_{1} A$, lying in $\partial H \backslash P$ and the other, $\partial_{2} A$, meeting $P$ in a single separating arc. Let $\rho, \sigma$, and $\tau$ be the components of $\partial P$ where $\partial_{2} A \cap \rho \neq \emptyset$ and $\partial_{1} A$ is parallel to $\sigma$.

Recall that $\partial$-compressing disks for $P$ in $H$ are product disks. There is a $\partial$-compressing disk $D_{\rho \sigma}$ for $P$ meeting $\rho$ and $\sigma$, and another one, $D_{\rho \tau}$, meeting $\rho$ and $\tau$. Furthermore, we may choose these disks to be disjoint from $\partial_{2} A$ in $P$. Since $\partial_{2} A$ is homotopic to $\sigma$ in $H,\left|\partial_{2} A \cdot D_{\sigma \rho}\right|=1$ and $\left|\partial_{2} A \cdot D_{\tau \rho}\right|=0$. However, there are no essential arcs in $\partial H \backslash P$ which connect $\rho$ to itself and have these intersection numbers. Therefore we may isotop $\partial_{2} A$ to lie entirely in $P$, and then we are in a case already dealt with.

4. If there is a pair of $\operatorname{arcs}$ of intersection of $P \cap P^{\prime}$ which are parallel in $P^{\prime}$, let $D$ be a subdisk of $P^{\prime}$ which is bounded by two such arcs whose interior is disjoint from $P$. If $D \subseteq H$, then $D$ must be inessential by Lemma 4.10, 
so we can reduce $\left|P \cap P^{\prime}\right|$. Therefore suppose that $D \subseteq J$ and that $D$ is an $(r, s)$ disk in the sense that it intersects $\partial_{1} P \cup \partial_{2} P$ in $r$ points and $\partial_{3} P$ in $s$ points ( $c f$. proof of Lemma 4.1). Then $r+s=4$. Suppose further that $(J, P)$ arises as the exterior of an $m / n$ rational tangle as in section 4 . Let $\bar{m}$ be the $\bmod n$ inverse of $-m$ such that $2|\bar{m}| \leq n$. Since $L$ is hyperbolic, $|\bar{m}|>1$. Note also that $r$ is even because $D \cap P$ consists of two parallel arcs. Therefore by [13, Lemma 3.4], $s \geq 4$, and so $D$ is a $(0,4)$ disk. However, this is impossible by [13, Lemma 2.3(1)].

5. If there are no parallel arcs of intersection of $P \cap P^{\prime}$ in $P$ or $P^{\prime}$, then $P \cap P^{\prime}$ consists of three mutually nonparallel arcs in both $P$ and $P^{\prime}$. We may choose a subdisk $D$ of $P^{\prime}$ bounded by these arcs such that $D \subseteq J$. In this case, $D$ is a $(4,2)$ disk. However, from the previous argument we know that $s \geq 4$, a contradiction.

Proposition 6.2. Let $L_{1}$ and $L_{2}$ be hyperbolic 2-bridge knots which are not mirror images, and let $p, q, p^{\prime}$, and $q^{\prime}$ be integers with $p<q, p^{\prime}<q^{\prime}, \operatorname{gcd}(p, q)=$ 1 , and $\operatorname{gcd}\left(p^{\prime}, q^{\prime}\right)=1$. Then there is a homeomorphism $M_{p, q}^{L_{1}} \rightarrow M_{p^{\prime}, q^{\prime}}^{L_{2}}$ taking $K_{p, q}^{L_{1}}$ to $K_{p^{\prime}, q^{\prime}}^{L_{2}}$ iff $L_{1}=L_{2}, p=p^{\prime}$, and $q=q^{\prime}$.

Proof. When $L$ is a hyperbolic 2-bridge knot, the triple of spaces obtained by attaching a 2-handle along each component of $\partial P^{L}$ is an invariant of $\left(M^{L}, K^{L}\right)$ by Lemma 6.1. After attaching a 2-handle along $\partial_{3} P^{L_{i}} \subseteq M^{L_{i}}$, we obtain $E\left(L_{i}\right), i=1,2$. Since the other two components of $\partial P^{L_{i}}$ are primitive, attaching a 2 -handle along them yields a solid torus.

First, focus on the 2-handle whose attaching curve is $\partial_{3} P^{L_{i}}$. A homeomorphism of $\left(M^{L_{1}}, K^{L_{1}}\right)$ with $\left(M^{L_{1}}, K^{L_{2}}\right)$ would extend across this 2-handle to give a homeomorphism of $E\left(L_{1}\right)$ with $E\left(L_{2}\right)$. However, these spaces are not homeomorphic unless $L_{1}=L_{2}[8]$.

Similarly, a homeomorphism of $\left(M_{p, q}^{L}, K_{p, q}^{L}\right)$ with $\left(M_{p^{\prime}, q^{\prime}}^{L}, K_{p^{\prime}, q^{\prime}}^{L}\right)$ would extend across a 2-handle attached along $\partial_{1} P$ or $\partial_{2} P$ to give homeomorphisms $h_{1}$ and $h_{2}$ of solid tori such that $h_{i}\left(K_{p, q}\right)=K_{p^{\prime}, q^{\prime}}, i=1,2$. By Lemma 4.2, we must have $p=p^{\prime}$ and $q=q^{\prime}$.

Proposition 6.3. The spaces $\left(M(\lambda), K^{\prime}\right)$ do not arise from the construction of section 4 .

Proof. From the proof of Proposition 6.2, we know that the triple of spaces obtained by attaching 2-handles along the components of $\partial P$ is an invariant of the spaces constructed in section 4. Furthermore, this invariant consists of a 2-bridge knot exterior and two solid tori. But from the proof of Proposition 5.2 we see that attaching 2-handles to $M(\lambda)$ along $\partial_{1} P$ yields the connect sum of a lens space of order $p$ and a solid torus. 


\section{$7 \quad$ The position of tunnels}

Fix a nontrivial 2-bridge knot $L$ and integers $0<p<q$ with $\operatorname{gcd}(p, q)=1$, and let $(M, K)=\left(M_{p, q}^{L}, K_{p, q}^{L}\right)$. Suppose that $K$ is 1 -bridge in $M$ so that there is a tunnel $t$ for $K$ as in Proposition 3.2. Then $M^{\prime}=\overline{M \backslash N(K \cup t)}$ is a handlebody of genus three. In this section we will isotop $t$ to lie in the handlebody $H$ while keeping $K \subseteq H$. Then we will isotop $t$ to be disjoint from the disk $E$ described in section 3 . This will allow us to make arguments about $\partial$-compressing disks for $P$ in $M^{\prime}$.

\subsection{The position of $t$ with respect to $P$}

First suppose that we have isotoped $t$ to minimize the number of intersections of $P$ and $t$ while keeping $K \subseteq H$. Define $P^{\prime}=P \cap M^{\prime}$ and let $M^{\prime}=H^{\prime} \cup_{P^{\prime}} J^{\prime}$ where $H^{\prime} \subseteq H$ and $J^{\prime} \subseteq J$. Note that $P^{\prime}$ is incompressible in $M^{\prime}$ since if it were not, we could find a compressing disk and use it to reduce $|P \cap t|$.

Let $D$ be a meridian disk for $M^{\prime}$, suppose that $|P \cap t|>0$, and suppose further that $D$ does not intersect $P^{\prime}$. Then we may isotop $D$ so that $\partial D \cap \partial N(t)$ is empty, and therefore $\partial D$ lies either on $\partial N(K), \partial J \backslash P$, or $\partial H \backslash P$. The first cannot happen by Proposition 4.9, and the second two are impossible because both of these surfaces are incompressible. Therefore either $t \cap P$ is empty or $D$ meets $P^{\prime}$.

Lemma 7.1. The intersection $t \cap P$ is empty.

Proof. We may isotop $D$ so that it intersects $P^{\prime}$ minimally and $D \cap P^{\prime}$ consists of arcs essential in $P^{\prime}$. An outermost $\operatorname{arc}$ in $D$ gives a disk $D^{\prime}$ with $\partial D^{\prime}=\alpha \cup \beta$, $\alpha \subseteq P^{\prime}$ essential and $\beta \subseteq \partial M^{\prime}$.

Call components of $\partial P^{\prime} \backslash \partial P$ new boundary components and components of $\partial P$ old. Then there are several cases:

1. The arc $\alpha$ connects two distinct old boundary components. Then we get a boundary compressing disk for $P$, which must lie in $H$ by Lemma 4.1. The boundary compression is disjoint from $K$. However, there are no $\partial$-compressing disks for $P$ in $H$ that do not meet $K$ by Lemma 4.8.

2. The arc $\alpha$ connects an old boundary component to itself. Suppose that $\alpha$ is nontrivial in $P$. Then we may isotop $\partial D^{\prime}$ so that $\partial D^{\prime} \cap \partial N(t)=\emptyset$. Therefore $D^{\prime}$ is a $\partial$-compressing disk for $P$ in $J$ or $H$, and since we know that $P$ is $\partial$-incompressible in $J, D^{\prime}$ must be a $\partial$-compressing disk for $P$ in $H$. However, $D^{\prime} \cap K=\emptyset$, contradicting Lemma 4.8.

If $\alpha$ is trivial in $P$, then $\beta$ must be inessential in $\overline{\partial J \backslash P}$ or $\overline{\partial H \backslash P}$ because otherwise we would obtain a compressing disk for this incompressible sur- 
face. But in this case we may isotop $D^{\prime}$ to be a compressing disk for the incompressible surface $P^{\prime}$.

3. The arc $\alpha$ connects an old and a new boundary component. Then $D^{\prime}$ guides an isotopy of $t$ reducing the number of intersections of $t$ with $P$ by one and leaving one endpoint of $t$ on the other side of $P$.

4. The arc $\alpha$ connects two new boundary components. Then $D^{\prime}$ guides an isotopy of $t$ reducing the number of intersections of $t$ with $P$ by two.

5. The arc $\alpha$ connects a new component to itself. If $\alpha$ extends to an inessential simple closed curve in $P$, then $\beta$ must be essential in $\overline{\partial H^{\prime} \backslash P^{\prime}}$ or $\overline{\partial J^{\prime} \backslash P^{\prime}}$ because otherwise we would get a compressing disk for $P^{\prime}$ by pushing $\beta$ to $P^{\prime}$. The disk $D^{\prime}$ extends to an annulus in $H$ or $J$, and we can cap off the boundary component containing $\alpha$ in $P$ (since this curve is inessential there) to get a compressing disk for $\overline{\partial J \backslash P}, \partial N(K)$, or $\overline{\partial H \backslash P}$. These are impossible by Lemma 4.1, Proposition 4.9, and the fact that $H \cong P \times I$, respectively.

So suppose that $\alpha$ extends to an essential simple closed curve in $P$. Then $D^{\prime}$ extends to an annulus $A$ in $N=J$ or $H$ with one boundary component, $\partial_{1} A$, on $P$, and the other, $\partial_{2} A$, on $\partial N$. The surface $A$ is incompressible because $\alpha$ extends to an essential simple closed curve in $P$. By Proposition $4.9, \partial_{2} A$ cannot lie on $\partial N(K)$. Therefore $\partial_{2} A$ is parallel to a component of $\partial P$ in $\partial N$. We may isotop $A$ so that $\partial A \subseteq P$ and use Lemma 4.6 to isotop $A$ through $P$, reducing $|t \cap P|$.

\subsection{The position of $t$ with respect to $E$}

By Lemma 7.1, we may take $t \cap P=\emptyset$. Let $E^{\prime}$ be the planar surface $E \cap H^{\prime}$. Note that $P$ is $\partial$-compressible in $H^{\prime}$ by Lemma 5.4 and Lemma 4.1.

Lemma 7.2. Let $D$ be a $\partial$-compressing disk for $P$ in $H^{\prime}$ which minimizes the tuple $\left(\left|D \cap E^{\prime}\right|,|D \cap \partial N(t)|,\left|\partial D \cap \partial E^{\prime} \cap P\right|\right)$.

- If $\partial D$ does not separate $P$, then $\partial D \cap \partial E^{\prime} \cap P=\emptyset$.

- If $\partial D \cap P$ connects $\partial_{1} P$ to itself, then $\left|\partial D \cap \partial E^{\prime} \cap P\right|=q-1$.

- If $\partial D \cap P$ connects $\partial_{2} P$ to itself, then $\left|\partial D \cap \partial E^{\prime} \cap P\right|=p-1$.

- If $\partial D \cap P$ connects $\partial_{3} P$ to itself, then $\left|\partial D \cap \partial E^{\prime} \cap P\right|=1$.

Proof. Recall that the arcs of intersection of $E^{\prime}$ and $P$ are nonseparating according to Lemma 4.4. The first claim follows from the fact that we can isotop nonseparating arcs in a 3 -punctured sphere to be disjoint. The isotopy pushes intersections of arcs in $P$ to intersections of arcs in $\overline{\partial H^{\prime} \backslash P}$ and can be chosen so that it does not increase $\left|D \cap E^{\prime}\right|,|\partial D \cap P|$, or $|\partial D \cap \partial N(t)|$. 
An essential separating arc meets a nonseparating arc in a pair of pants minimally zero or one times. Recall that $\partial E \cap P$ consists of a single arc connecting $\partial_{1} P$ and $\partial_{2} P, p-1$ arcs connecting $\partial_{1} P$ and $\partial_{3} P$, and $q-1$ arcs connecting $\partial_{2} P$ and $\partial_{3} P$. Therefore we may isotop $D$ to one of the forms above. This isotopy pushes intersections of arcs in $P$ to intersections of arcs in $\partial H^{\prime} \backslash P$ and can be chosen so that it does not increase $\left|D \cap E^{\prime}\right|,|\partial D \cap P|$, or $|\partial D \cap \partial N(t)|$.

Let $D$ be a $\partial$-compressing disk for $P$ in $H^{\prime}$ which meets $\partial N(t)$. Denote by $\epsilon$ the subarc of $\partial D$ such that $\epsilon \cap P \neq \emptyset, \partial \epsilon \subseteq \partial N(t)$, and int $\epsilon \cap \partial N(t)=\emptyset$. This is a proper subarc of $\partial D$ by Lemma 4.8. It is the largest subarc of $\partial D$ lying in $\partial H$ which contains $P \cap \partial D$.

Lemma 7.3. We can isotop $t$ so that $t \cap E=\emptyset$. During the isotopy, one foot of $t$ lies in $\partial H \backslash P$.

Proof. Isotop $t$ to intersect $E$ minimally, recall that we defined $E^{\prime}=E \cap H^{\prime}$, and let $D$ be a $\partial$-compressing disk for $P$ in $H^{\prime}$ minimizing $\left(\left|D \cap E^{\prime}\right|, \mid D \cap\right.$ $\left.\partial N(t)|,| \partial D \cap \partial E^{\prime} \cap P \mid\right)$. If $D \cap \partial N(t)=\emptyset$, then $\partial D$ lies on $\partial N(K)$ or $\partial H$. It cannot lie on $\partial N(K)$ since $D$ is a $\partial$-compressing disk for $P$. If $\partial D \subseteq H$, then $D$ gives a disk $D^{\prime}$ in $H$ which is a $\partial$-compressing disk for $P$ but does not meet $K$. This is impossible by Lemma 4.8 , and therefore $D \cap E^{\prime} \neq \emptyset$.

By a standard innermost circle argument we may assume that $D \cap E^{\prime}$ consists of arcs; let $\alpha$ be such an arc outermost in $D$.

Suppose that $\alpha$ cuts off a subdisk $D^{\prime}$ of $D$ with $\partial D^{\prime}=\alpha \cup \beta$ where $\beta \subseteq \partial H$ and $\beta \cap P=\emptyset$. Then $\alpha$ is a properly embedded arc in $E$, and we can surger $E$ along $D^{\prime}$ to obtain two new disks $E_{1}$ and $E_{2}$ in $H$. If $\partial E_{i}$ meets $P$, then by considering the algebraic intersection of $\partial E_{i}$ and $\partial P$ we see that $E_{i}$ is essential. If $\partial E_{i}$ is disjoint from $\partial P$ then $E_{i}$ is trivial because $\partial H \backslash P$ is incompressible. We can then isotop $D$ to reduce $D \cap E^{\prime}$ while keeping $D$ a $\partial$-compressing disk for $P$. Therefore both $E_{1}$ and $E_{2}$ are essential in $H$. One of these new disks, say $E_{1}$, must be nonseparating since $E$ is. By Lemma $4.3, E_{1}$ is isotopic to $E$. However, by Lemma $4.10, E_{2}$ meets $P$ in at least three arcs, and therefore $E_{1}$ violates Lemma 4.4 .

Suppose that $\alpha$, seen as an arc in $D$, has both endpoints in the same subarc of $\partial N(t) \cap \partial D$. Then $\alpha$ cuts off a subdisk $D^{\prime}$ of $D$ which guides an isotopy of $t$ through $E$ to reduce $|t \cap E|$ by two. Note that the foot of $t$ on $\partial H$ does not meet $P$ during this isotopy.

Suppose that $\alpha$ has one endpoint in an arc of $\partial N(t) \cap \partial D$ and the other in an adjacent arc of $\partial H \cap \partial D$ so that it cuts of a subdisk $D^{\prime}$ of $D$ which is disjoint from $P$. Then $D^{\prime}$ guides an isotopy of $t$ which reduces $|t \cap E|$ by one. The foot of $t$ on $\partial H$ does not meet $P$ during this isotopy.

Suppose that $\alpha$ has one endpoint in an arc of $\partial N(t) \cap \partial D$ and the other endpoint in a different arc of $\partial N(t) \cap \partial D$ so that $\alpha$ cuts off a subdisk $D^{\prime}$ of $D$ which does not meet $P$. The disk $D^{\prime}$ extends to an embedded annulus $A$ in $H$ with one 
boundary component on $E$ and the other on either $\partial N(K)$ or $\partial H$. We may cap off the boundary component of $A$ on $E$ to obtain a disk $D^{\prime \prime}$ with either $\partial D^{\prime \prime} \subseteq \partial N(K)$ or $\partial D^{\prime \prime} \subseteq \partial H$. Note that $\partial D^{\prime \prime}$ must be essential on $\partial H$ or $\partial N(K)$ since otherwise we could reduce $|\partial D \cap \partial N(t)|$. No essential curve on $\partial N(K)$ bounds a disk in $\overline{H \backslash N(K)}$, so $\partial D^{\prime \prime}$ must lie on $\partial H$. In this case, note that $\partial D^{\prime \prime} \cap P=\emptyset$ and $D^{\prime \prime} \cap K=\emptyset$. But we have already seen that disks which do not meet $K$ must meet $P$. Therefore we may rule out such arcs.

Finally, note that since $t \cap E \neq \emptyset$, every component of $\partial N(t) \cap \partial D$ contains the endpoint of at least one arc of $D \cap E^{\prime}$. Therefore there must be an arc of $D \cap E^{\prime}$ which is outermost in $D$ and has one of the forms above unless every arc meeting $\partial N(t)$ has its other endpoint in $P$. So suppose that this is the case, and recall that $\epsilon$ is the largest subarc of $\partial D$ which lies in $\partial H$ and meets $P$. Since $\epsilon \cap \partial E^{\prime} \cap P \neq \emptyset, D$ must separate $P$ by Lemma 7.2. By the minimality of $\partial D \cap \partial E \cap P$, we know exactly where the endpoints of $\epsilon$ lie on $\partial P$; the possibilities are shown in Figure 6 (cf. Figure 4).

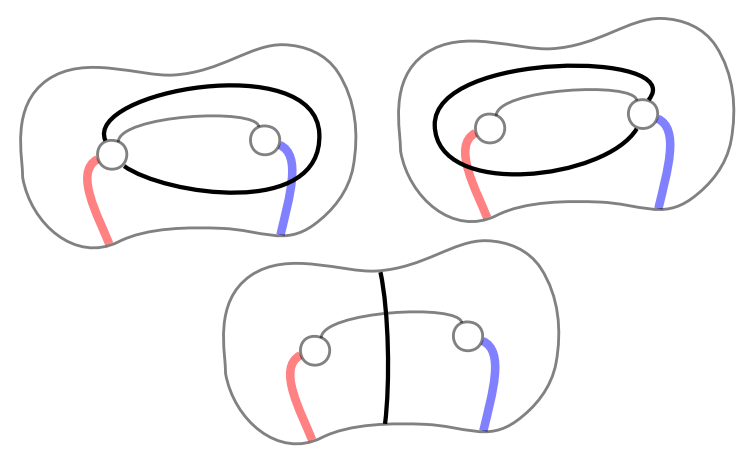

Figure 6: The possibilities for $\partial D \cap P$ when $\partial D$ is separating (bold)

Examining Figure 4 we see that $\epsilon \cap R \cap \partial E \neq \emptyset$. But in this case there is an arc of intersection, outermost in $D$, which connects $P$ to an adjacent $\operatorname{arc}$ of $\partial H$. This arc cuts off a subdisk $D^{\prime}$ of $D$ whose interior does not meet $E \subseteq H$. We may surger $E$ along $D^{\prime}$ to obtain two disks $E_{1}$ and $E_{2}$ in $H$. By considering the algebraic intersection of $\partial E_{i}$ and $\partial P$ we see that $E_{i}$ is essential, $i=1,2$. One of these, say $E_{1}$, must be nonseparating since $E$ is. By Lemma $4.3, E_{1}$ is isotopic to $E$. However, by Lemma $4.10, E_{2}$ meets $P$ in at least three arcs, and therefore $E_{1}$ violates Lemma 4.4 .

\section{Boundary compressing disks for $P$ in $H^{\prime}$}

In this section we assume the existence of a tunnel $t$ as in Proposition 3.2 so that $M^{\prime}=\overline{M \backslash N(K \cup t)}$ is a handlebody of genus three. We may assume that $H^{\prime}=\overline{H \backslash N(K \cup t)}$ is a handlebody of genus 3 by Lemma 7.1. Because 
it is not $\partial$-compressible in $J$, the surface $P \subseteq M^{\prime}$ is $\partial$-compressible in $H^{\prime}$ by Lemma 5.4. We want to show that $\partial$-compressing along any $\partial$-compressing disk for $P$ yields an annulus whose core is not primitive in either $H^{\prime}$ or $J$, contradicting Proposition 5.5 and showing that $K$ cannot be 1-bridge.

Lemma 7.3 shows that the nonseparating disk $E$ which is disjoint from $K$ in $H$ is also an essential disk in $H^{\prime}$. We consider first the case when there is a $\partial$-compressing disk disjoint from $E$.

\subsection{Boundary compressing disks disjoint from $E$}

Let $D$ be a $\partial$-compressing disk for $P$ in $H^{\prime}$ such that $D \cap E=\emptyset$. Choose $D$ to minimize $(|\partial D \cap \partial N(t)|,|\partial D \cap \partial E \cap P|)$. Note that $D$ must be nonseparating in $P$ since a separating disk necessarily meets $E$ in $P$. If $D$ does not meet $\partial N(K)$, then we may isotop $\partial D$ so that it lies entirely in $\partial H$. Thus $D$ gives a disk in $H$ with $D \cap K$ empty and $D \cap P$ consisting of one essential arc, which is impossible by Lemma 4.8 .

Lemma 8.1. Suppose that $D$ is a boundary compressing disk for $P$ in $H^{\prime}$ such that $D \cap E=\emptyset$. Then we may isotop $D$ so that $D \cap E$ is still empty and $\partial D \cap \partial N(t)$ consists of two arcs.

Proof. Suppose the endpoint of the tunnel is in region $R_{t}$ of $R \backslash \partial E$. Then $R_{t} \cap H^{\prime} \cap \partial D$ consists of a number of arcs, exactly two of which meet $\partial R_{t}$. Recall that every component of $R \backslash \partial E$ is a disk. Therefore if there are more than two arcs, there is a trivial arc of $R_{t} \cap H^{\prime} \cap \partial D$ which we can use to reduce $|\partial D \cap \partial N(t)|$.

Lemma 8.2. Let $D$ be a $\partial$-compressing disk for $P$ disjoint from $E$. Then the core of the annulus obtained by $\partial$-compressing $P$ along $D$ is not primitive in either $J$ or $H^{\prime}$.

Proof. By Lemma 8.1, the boundary of any $\partial$-compressing disk $D$ disjoint from $E$ must contain a single arc in $P$ and two arcs connecting $\partial P$ to the tunnel. It follows that there is a region of $P$ whose boundary meets some region of $R$ in two distinct subarcs.

If a rectangular region of $P$ is attached along $\partial P$ to a region of $R$, we see that $\partial E$ is disconnected. The same holds if a rectangular region of $R$ is attached along $\partial P$ to a region of $P$. Therefore $\partial D \cap P$ and $\partial D \cap R$ lie in the hexagonal regions, and it follows from examining Figure 4 that $\partial D \cap \partial_{3} P=\emptyset$.

By Lemma 8.1, the disk $D$ extends to an annulus $A$ embedded in $\overline{H \backslash N(K)}$ with one boundary component, $\partial_{1} A$, on $\partial H$, and the other, $\partial_{2} A$, on $\partial N(K)$. Furthermore, $\partial_{1} A$ meets $P$ in an arc connecting $\partial_{1} P$ and $\partial_{2} P$. Let $\alpha$ be the core of the annulus obtained by $\partial$-compressing $P$ in $H^{\prime}$, so that $\alpha$ is parallel to $\partial_{3} P$. This curve is not primitive in $J$ by construction. 
Attaching a 2-handle to $H$ along $\alpha$ we obtain a solid torus $S$ containing a knot $K$. The space $\overline{S \backslash N(K)}$ is irreducible by Lemma 4.2. By [4, Lemma 2.5.3], $\partial_{2} A$ is either meridional on $\partial N(K)$ or meets a meridian of $\partial N(K)$ exactly once. The latter case is impossible since $K$ is not isotopic to $\partial S$ by Corollary 5.3. Therefore $A$ extends to a meridian disk $D^{\prime}$ of $S$ meeting $K$ exactly once, which shows that $K$ is the connect sum of the core curve of $S$ with a nontrivial knot. Therefore the exterior of $K \cup t$ in $S$ is homeomorphic to a nontrivial knot exterior with a 1-handle attached, and so $\alpha$ is not primitive in $H^{\prime}$.

\subsection{Boundary compressing disks meeting $E$}

In this section, let $D$ be a $\partial$-compressing disk for $P$ in $H^{\prime}$ which minimizes $(|D \cap E|,|D \cap \partial N(t)|,|\partial D \cap \partial E \cap P|)$ and suppose that $|D \cap E|>0$. We may assume that every component of $D \cap E$ is an arc. There are several types of "forbidden" $\operatorname{arcs}$ in $D$ and $E$ which we will use to argue about the existence of $\partial$-compressing disks for $P$ in $H^{\prime}$ :

Lemma 8.3. Let $\alpha$ be a component of $D \cap E$. Then either

- $\alpha$ connects two components of $\partial E \backslash P$ and separates $E$ into two subdisks each containing at least two components of $\partial E \cap P$ on their boundary, or

- $\alpha$ connects a component of $\partial E \cap P$ with a nonadjacent component of $\partial E \backslash P$.

Proof. Let $\alpha$ be an arc of intersection of $D \cap E$ and suppose that $\alpha$ either has both endpoints in the same component of $\partial E \backslash P$ or both endpoints in the same component of $\partial E \cap P$. We may assume $\alpha$ is an outermost such arc in $E$ which cuts off a disk $E^{\prime}$ whose interior does not meet $D$. Then we may surger $D$ along $E^{\prime}$ to obtain a new $\partial$-compressing disk meeting $E$ fewer times.

Suppose that $\alpha$ has each endpoint in a different component of $\partial E \cap P$. Among all arcs in $D$ with endpoints on $\partial D \cap P$ choose an outermost one $\alpha^{\prime}$ so that $\alpha^{\prime}$ cuts off a subdisk $D^{\prime}$ of $D$ such that the interior of $D^{\prime}$ does not meet $E$. We may assume that $\alpha^{\prime}$ does not have both endpoints in the same component of $\partial E \cap P$ in $E$ since if it did, we could find a simpler $\partial$-compressing disk as in the previous paragraph. Surgering $E$ along $D^{\prime}$ we obtain a nonseparating disk which must be isotopic to $E$ by Lemma 4.3. However, this disk contradicts Lemma 4.4.

Suppose then that $\alpha$ has one endpoint in a component of $\partial E \cap P$ and the other in an adjacent component of $\partial E \backslash P$. We may assume that $\alpha$ is outermost in $E$ and cuts off a subdisk $E^{\prime}$ of $E$ whose interior does not meet $D$. By Lemma 7.2, $\partial D$ separates $P$. We may surger $D$ along $E^{\prime}$ to obtain a new disk $D^{\prime}$ which meets $P$ in a single arc. Since $\partial D$ separates $P$ and no $\operatorname{arc}$ of $\partial E \cap P$ is separating, $\partial D^{\prime} \cap P$ is essential in $P$. Therefore $D^{\prime}$ is a new $\partial$-compressing disk which meets $E$ fewer times.

Finally, suppose that $\alpha$ has both endpoints on $\partial E \backslash P$ and cuts off a disk $E^{\prime}$ containing exactly one arc of $\partial E \cap P$. We may assume that $\alpha$ is outermost in 
$E$ so that it cuts off a subdisk $E^{\prime}$ whose interior is disjoint from $D$. As above, we can surger $D$ along $E^{\prime}$ to obtain a new $\partial$-compressing disk which has fewer intersections with $E$.

Recall that the arc $\epsilon$ is defined as the largest subarc of $\partial D$ lying in $\partial H$ and meeting $P$. Let $\Delta=\partial D \backslash(\epsilon \cup \partial N(K \cup t))$.

Lemma 8.4. There are no arcs of $D \cap E$ which have both endpoints in $\epsilon$. There are no arcs of $D \cap E$ which have both endpoints in the same component of $\Delta$.

Proof. There are no arcs with both endpoints in $\epsilon \cap P$ by Lemma 8.3. Let $\alpha$ be a component of $D \cap E$ which has both endpoints in $\epsilon$. We may assume that $\alpha$ is outermost in $D$ and cuts off a subdisk $D^{\prime}$ with $\partial D^{\prime}=\alpha \cup \beta, \beta \subseteq \epsilon$.

If $\alpha$ has one endpoint in $\epsilon \cap P$ and the other in $\epsilon \cap R$, we may surger $E$ along $D^{\prime}$ to obtain a nonseparating disk which must be isotopic to $E$ by Lemma 4.3. By Lemma 8.3 this disk meets $P$ fewer than $p+q-1$ times, and so it contradicts Lemma 4.4. The same conclusion holds if $\alpha$ has both endpoints in $\epsilon \cap R$.

If $\alpha$ has both endpoints in a component $\delta$ of $\Delta$, we may assume that $\alpha$ is outermost in $D$ and cuts off a subdisk $D^{\prime}$ with $\partial D^{\prime}=\alpha \cup \beta, \beta \subseteq \delta$. Surgering $E$ along $D^{\prime}$ gives a disk violating Lemma 4.4 as above.

Note that Lemma 8.4 shows that $\Delta$ is nonempty. Call two distinct components $\delta_{1}$ and $\delta_{2}$ of $\Delta \cup\{\epsilon\}$ adjacent if there is a component $\phi$ of $\partial D \cap \partial N(K \cup t)$ such that $\phi$ shares one endpoint with $\delta_{1}$ and the other with $\delta_{2}$. Orient $\partial D$ so that each component of $\Delta$ inherits an orientation. The label sequence of a component $\delta$ of $\Delta$ is the ordered sequence of $\operatorname{arcs} \partial E \cap R$ that $\delta$ meets. The reverse of a label sequence $x_{1}, x_{2}, \ldots, x_{n}$ is $x_{n}, x_{n-1}, \ldots, x_{1}$.

Let $\delta$ be a component of $\Delta$. Then $\delta$ extends to a simple closed curve $\delta^{\prime}$ in $R$ which is parallel to a component $\gamma$ of $\partial P$ by minimality of $(|D \cap E|,|\partial D \cap \partial N(t)|)$. By abuse of notation, we say that $\delta$ is parallel to $\gamma$.

Lemma 8.5. All components of $\Delta$ are parallel on $R$, and no component of $\Delta$ is parallel to $\partial_{3} P$.

Proof. Suppose that there are two components $\delta_{1}$ and $\delta_{2}$ whose label sequences are different and not the reverse of one another. By minimality of $|D \cap E|, \delta_{1}$ and $\delta_{2}$ must be parallel to different components of $\partial P$. Therefore $\partial D$ meets every component of $\partial E \cap R$. But an arc of $D \cap E$, outermost in $E$, must then violate Lemma 8.3.

If there is a component of $\Delta$ which is parallel to $\partial_{3} P$, then every arc of $\partial E \cap R$ except for $c$ contains the endpoint of an arc of $D \cap E$. Since there are at least two outermost $\operatorname{arcs}$ of $D \cap E$ in $E$, there is at least one which violates Lemma 8.3. 
Let $D^{\prime}$ be a $2 n$-gon, $n>1$, in $D$ whose boundary consists of a union of components of $\partial D \backslash \partial E$ and components of $D \cap E$ and whose interior is disjoint from $E$. At each vertex of $D^{\prime}$ we see a label $a^{P}, b_{i}^{W}, c^{R}$, or $d_{l}^{W}$, where $W=R$ or $P$. Suppose that the vertex set of $D^{\prime}$ contains exactly two labels $x$ and $y$, and suppose further that as we go around $\partial D^{\prime}$ these labels alternate. We call $\partial D^{\prime}$ a Scharlemann cycle. An example is shown in Figure 7. The bold arcs represent $\partial D \cap \partial N(K \cup t)$ and the shaded region is $D^{\prime}$. Note that since the vertex set of $D^{\prime}$ contains exactly two labels and there are no arcs of $D \cap E$ with both endpoints in $\partial D \cap P$, we have $\partial D^{\prime} \cap P=\emptyset$.

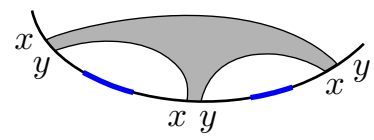

Figure 7: A Scharlemann cycle appearing in $D$

Note that the arcs of $\partial D^{\prime} \cap E$ are parallel in $E$, and the arcs of $\partial D^{\prime} \cap \partial D$ are parallel in $R$. Let $A_{E}$ be a subdisk of $E$ whose boundary consists of two subarcs of $\partial E$ and two components of $\partial D^{\prime} \cap E$ such that $A_{E}$ contains all components of $\partial D \cap E$. Similarly, let $A_{R}$ be a subdisk of $R$ whose boundary consists of two subarcs of $\partial E$ and two components of $\partial D^{\prime} \cap \partial D$ such that $A_{R}$ contains all components of $\partial D^{\prime} \cap \partial D$. Then $S=N\left(A_{E} \cup A_{R}\right)$ is a solid torus in $H^{\prime}$. Since $n>1, N\left(S \cup D^{\prime}\right)$ is a punctured lens space. (We can see this by noting that $N\left(S \cup D^{\prime}\right)$ is obtained by attaching the 2 -handle $N\left(D^{\prime}\right)$ to a solid torus so that the attaching curve meets a meridian algebraically more than once.)

In particular, $H^{\prime}$ is reducible, which is impossible since we are assuming that $H^{\prime}$ is a handlebody. Therefore such disks $D^{\prime}$ do not exist in $D$. We can use this criterion to show:

Lemma 8.6. Let $\delta_{1}$ and $\delta_{2}$ be adjacent components of $\Delta$, and suppose that $\delta_{1}$ has label sequence $x_{1}, \ldots, x_{n}$. Then there are at most $\lfloor n / 2\rfloor$ arcs of $D \cap E$ with one endpoint in $\delta_{1}$ and the other in $\delta_{2}$.

Proof. Suppose not. Then there are two adjacent components $\delta_{1}$ and $\delta_{2}$ of $\Delta$ with more than $\lfloor n / 2\rfloor$ arcs of $D \cap E$ connecting them. By Lemma 8.4 there are no arcs with both endpoints in $\delta_{i}, i=1,2$, and therefore these arcs are all parallel in $D$. By Lemma 8.5, $\delta_{2}$ has the label sequence of $\delta_{1}$ or its reverse.

If $\delta_{2}$ has the reverse label sequence of $\delta_{1}$, then there is an arc both of whose endpoints have the same label. This contradicts Lemma 8.3.

So suppose that $\delta_{2}$ has the same label sequence as $\delta_{1}$. If $n$ is odd, there is an arc both of whose endpoints have label $x_{(n+1) / 2}$, and this arc violates Lemma 8.3. On the other hand, if $n$ is even, then there is a Scharlemann cycle with label set $\left\{x_{n / 2}, x_{n / 2+1}\right\}$. 
Lemma 8.7. There are no arcs of $D \cap E$ with endpoints in nonadjacent components of $\Delta$.

Proof. By Lemma 8.5, every component of $\Delta$ has the label sequence of $\delta$ or its reverse. Suppose there were an arc of $D \cap E$ with endpoints in nonadjacent components of $\Delta$, and choose $\alpha$ to be one outermost in $D$. The $\operatorname{arc} \alpha$ cuts off a subdisk $D^{\prime}$ in which every arc int $D^{\prime} \cap E$ connects adjacent components of $\Delta^{\prime}=\Delta \cap \partial D^{\prime}$.

If two components of $\Delta^{\prime}$ have label sequences which are the reverse of one another, then we can find two such components $\delta_{1}$ and $\delta_{2}$ which are adjacent. Suppose there is an arc of int $D^{\prime} \cap E$ connecting them. Then an outermost such arc in $D^{\prime}$ violates Lemma 8.3. If no $\operatorname{arcs}$ of int $D^{\prime} \cap E$ connect $\delta_{1}$ and $\delta_{2}$, then there is a component $\delta_{3}$ of $\Delta^{\prime}$ adjacent to $\delta_{1}$ such that every arc with one endpoint in $\delta_{1}$ has its other endpoint in $\delta_{3}$. This contradicts Lemma 8.6.

Therefore all components of $\Delta^{\prime}$ which are also components of $\Delta$ have the same label sequence; let $n$ be the length of this sequence. By Lemma 8.6, $n$ must be even and every component of $\Delta^{\prime}$ must contain exactly $n / 2$ parallel arcs connecting an adjacent component. But in this case, the $2\left|\Delta^{\prime}\right|$-gon which appears in $D^{\prime}$ is a Scharlemann cycle. This is impossible by the remarks following Lemma 8.5.

Lemma 8.8. Let $\delta$ be a component of $\Delta$ with label sequence $x_{1}, x_{2}, \ldots, x_{n}$. Then there are at least $2\lceil n / 2\rceil$ endpoints of arcs of $D \cap E$ in $\epsilon$.

Proof. No arcs of $D \cap E$ connect nonadjacent components of $\Delta$ by Lemma 8.7. Since at most $\lfloor n / 2\rfloor$ arcs connect adjacent components by Lemma 8.6, there must be at least $2\lceil n / 2\rceil$ arcs which have one endpoint in $\epsilon$.

Lemma 8.9. The arc $\epsilon$ meets $\partial E$ in $R$.

Proof. Suppose first that $\partial D$ is nonseparating in $P$ so that $\partial D \cap \partial E \cap P=\emptyset$. Then $\partial E \cap R \neq \emptyset$ by Lemma 8.8 .

If $\partial D$ separates $P$, then by minimality of $|\partial D \cap \partial E \cap P|$, we know exactly in which regions of $R$ the points $\epsilon \cap \partial P$ lie. The three cases are pictured in Figure 6, and we see that the endpoints of $\epsilon \cap P$ lie in different regions of $R$. Therefore $\epsilon$ must cross $\partial E$ in $R$.

Recall that we are assuming $1<p<q$.

Lemma 8.10. Let $\delta$ be a component of $\Delta$. Then there are at least $p$ arcs of intersection $D \cap E$ with endpoints in $\delta$.

Proof. By the previous remarks, $\delta$ is parallel to a component of $\partial P$, and these meet $E$ minimally $p$ times. 
Lemma 8.11. There is an arc $\alpha$ of $D \cap E$, outermost in $D$, with one endpoint in $\epsilon$ and the other in an adjacent component of $\Delta$. This arc cuts off a disk $D^{\prime}$ of $D$ with $\partial D^{\prime}=\alpha \cup \beta$. The endpoints of $\beta$ lie in $d_{\bar{q}}^{R}$ and $b_{\bar{p}}^{R}$ or $d_{(p-1) \bar{q}}^{R}$ and $b_{(q-1) \bar{p}}^{R}$ depending on whether the foot of the tunnel lies in $\mathrm{H}_{1}$ or $\mathrm{H}_{2}$. Furthermore, $\beta$ does not meet $P$.

Proof. The first part follows from Lemma 8.4 and Lemma 8.9. If $\alpha$ did not have an endpoint in a component of $\Delta$ adjacent to $\epsilon$ it could not be outermost by Lemma 8.10 .

If $\beta \cap P \neq \emptyset$, there is at least one other arc of $D \cap E$ with an endpoint in $\epsilon \cap R$ by Lemma 8.8. An outermost such arc gives the desired $\alpha$ by Lemma 8.6 and Lemma 8.7.

If $\beta$ has endpoints on the same component of $\partial E \cap R$ in $R$, we obtain an arc in $E$ contradicting Lemma 8.3. Furthermore, since $\alpha$ is outermost in $D$, we may surger $E$ along a subdisk of $D$ to obtain a new disk which does not meet $E$. Therefore $\beta$ has opposite signs of intersection with $\partial E$ in $R$.

Orienting $\partial E$ we see that all arcs of $\partial E \cap R$ in $R$ which connect two boundary components are coherently oriented. Therefore $\beta$ must lie in one of the hexagonal regions. Checking the possibilities, we see that the condition that $\beta$ has opposite signs of intersection with $\partial E$ implies that it meets either $d_{\bar{q}}^{R}$ and $b_{\bar{p}}^{R}$ or $d_{(p-1) \bar{q}}^{R}$ and $b_{(q-1) \bar{p}}^{R}$.

\section{Families of knots which are not 1-bridge}

Fix a nontrivial 2-bridge knot $L$. Let $q=p+1$ and write $(M, H, K)=$ $\left(M_{p, p+1}^{L}, H_{p, p+1}^{L}, K_{p, p+1}^{L}\right)$. Suppose that there is a tunnel $t$ as in Proposition 3.2 so that $M^{\prime}=\overline{M \backslash(K \cup t)}$ is a handlebody, and suppose that $t$ lies entirely in $H$.

Lemma 9.1. Any $\partial$-compressing disk for $P$ in $M^{\prime}$ violates Proposition 5.5.

Proof. We only need to consider $\partial$-compressing disks for $P$ in $H^{\prime}=\overline{H \backslash N(K \cup t)}$ which meet $E$ by Lemma 8.2. So let $D$ be a $\partial$-compressing disk for $P$ in $H^{\prime}$ which minimizes $(|D \cap E|,|D \cap \partial N(t)|,|\partial D \cap \partial E \cap P|)$, and let $D^{\prime}$ be as in Lemma 8.11. Examining the options given by that lemma, we see that $\alpha$ must either have one endpoint in $b_{-1}$ and the other in $d_{1}$ or one endpoint in $b_{1}$ and the other in $d_{-1}$. An arc with endpoints in $b_{1}$ and $d_{-1}$ violates Lemma 8.3, so $\alpha$ must be of the first type.

The $\operatorname{arc} \alpha$ cuts off a subdisk $E^{\prime}$ of $E$ which meets $P$ in exactly two $\operatorname{arcs}\left(b_{-1}^{P}\right.$ and $\left.d_{1}^{P}\right)$. An arc in $E^{\prime}$ with one endpoint in $c$ would violate Lemma 8.3, and so no component $\delta$ of $\Delta$ meets $c$. Therefore every component of $\Delta$ is parallel to $\partial_{3} P$, but this contradicts Lemma 8.5. 
Now we tackle the more difficult case when $q=2 p \pm 1, p>1$, and $q>3$. The intersection patterns of $\partial E \cap P$ and $\partial E \cap R$ are special in these cases, and they are pictured in Figure 8. The disk $E$ remains as in Figure 3. There are two hexagonal regions in each of $P$ and $R$; we will refer to these regions as $H_{1}^{P}, H_{2}^{P}$, $H_{1}^{R}$, and $H_{2}^{R}$. Note that when $q=2 p-1, H_{1}$ and $H_{2}$ are reversed from Figure 4 .

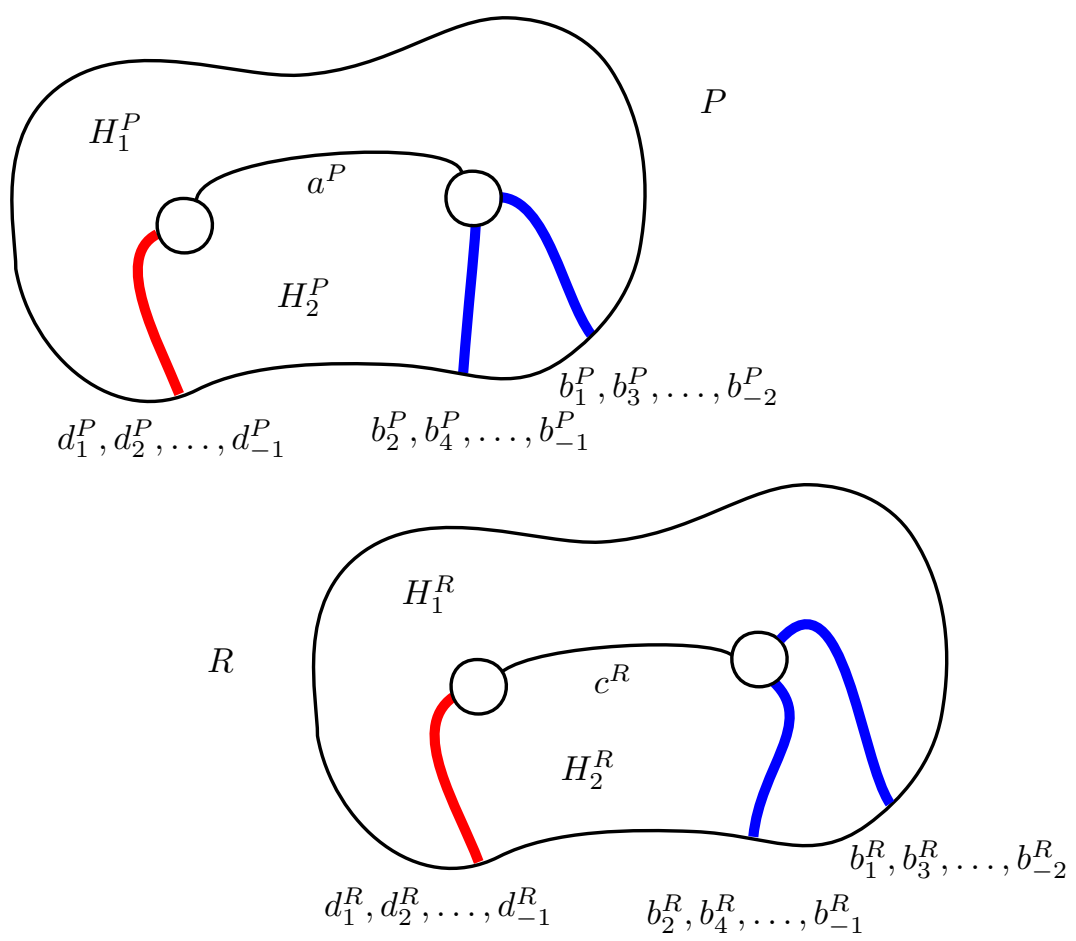

Figure 8: The surfaces $P$ and $R$ in the case $q=2 p \pm 1$

As before, let $D$ be a $\partial$-compressing disk for $P$ in $H^{\prime}$ which minimizes $(\mid D \cap$ $E||, D \cap \partial N(t)|,| \partial D \cap \partial E \cap P \mid)$. Let $\beta$ be as in Lemma 8.11. Examining the possibilities given by that lemma, we see that $\beta$ must have endpoints on $d_{-1}$ and $b_{2}$, or $d_{1}$ and $b_{-2}$. The two possibilities for $\alpha \subseteq E$ are shown in Figure 9 .

Let $R^{\prime}=R \cap H^{\prime}$.

Lemma 9.2. When $q=2 p \pm 1$, the arc $\epsilon$ has endpoints in different components of $R^{\prime} \backslash \Delta$.

Proof. Suppose that $\epsilon$ has its endpoints in the same component of $R^{\prime} \backslash \Delta$. Then $D$ extends to a properly embedded surface $S$ in $\overline{H \backslash N(K)}$ by attaching bands along $\partial D$. This surface has $|\Delta|+1$ boundary components on $\partial H,|\Delta|$ of which are parallel to a component of $\partial P$ and one of which meets $P$ in a single essential arc. Note that since the bands are attached in an orientation preserving way, $S$ is 


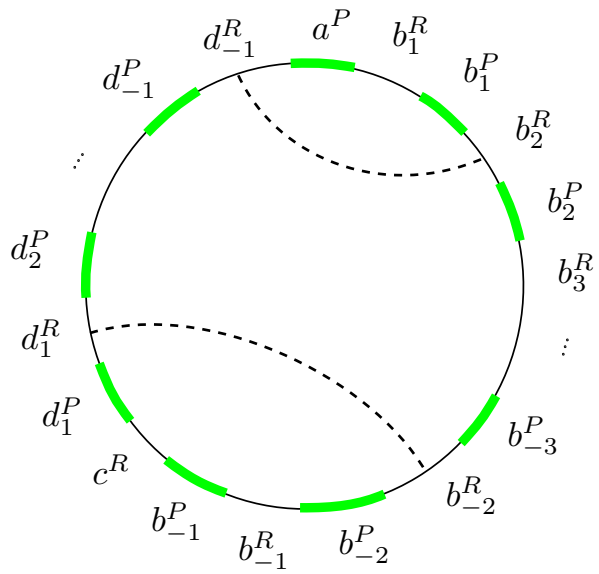

Figure 9: Possibilities for $\alpha$ in $E$

orientable. Furthermore, we can see that $S$ is a punctured sphere by calculating $\chi(S)$.

The surface $S$ has $|\Delta|$ boundary components parallel to a component $\partial P$. Let $\gamma$ be a simple closed curve in $R$ which does not meet $\epsilon$ and is parallel to this component. Note that $\gamma$ cannot be parallel to $\partial_{3} P$ by Lemma 8.5. After attaching a 2-handle to $H$ along $\gamma, S$ extends to an annulus $A$ in a solid torus $U$. Let $Q$ be the annulus in $\partial U$ consisting of $P$ together with the trivial disk bounded by the component of $\partial P$ which was made trivial by the 2 -handle attachment. Note that $Q$ is longitudinal on $\partial U$. One component of $\partial A$ lies on $\partial U$. Call it $\partial_{1} A$. The other, $\partial_{2} A$, lies on $\partial N(K)$.

We want to show that either $\partial_{1} A$ is parallel to a component of $\partial Q$ or meets $Q$ in an essential arc. If $\partial D$ is nonseparating in $P$, then $\partial_{1} A$ meets $Q$ in an essential arc, and so it is certainly nontrivial in $\partial U$. So suppose that $\partial D$ separates $P$. The possibilities for $\partial D \cap P$ appear in Figure 6. If $\epsilon$ meets the component of $\partial P$ parallel to $\gamma$, it is clear that we can isotop $\partial_{1} A$ to be parallel to a component of $\partial Q$. Using this fact, there are only a few remaining cases to check:

1. If $\partial D$ meets $\partial_{3} P$ and $\gamma$ is parallel to $\partial_{1} P$, then $\epsilon$ meets either $b_{2}^{R}$ or $b_{-2}^{R}$ by Lemma 8.11, depending on whether the tunnel exits in $H_{2}^{R}$ or $H_{1}^{R}$. In either case, the minimality of $|D \cap E|$ implies that $\partial D$ meets every component of $\partial E \cap R$. An outermost arc of $D \cap E$ in $E$ must then violate Lemma 8.3. If $\partial D$ meets $\partial_{3} P$ and $\gamma$ is parallel to $\partial_{2} P$, then $\epsilon$ meets either $d_{1}^{R}$ or $d_{-1}^{R}$ and the same argument applies.

2. If $\partial D$ meets $\partial_{2} P$, we see from Figure 6 that $\epsilon$ meets the components of $\partial_{2} P \backslash \partial E$ which lie on either side of $b_{1}^{R}$. If $\gamma$ is parallel to $\partial_{1} P$ and the tunnel exits in $H_{2}^{R}$, then $\epsilon$ meets $b_{2}^{R}, b_{4}^{R}, \ldots, b_{-1}^{R}$. If $\epsilon$ meets $b_{1}^{R}$, it is easy to see from Figure 9 that there is an arc forbidden by Lemma 8.3. However, if $\epsilon$ does not meet $b_{1}^{R}$, then $\partial D$ meets every arc of $\partial E \cap R$ except 
for $b_{1}^{R}$, and again there must be an outermost arc of $D \cap E$ in $E$ which violates Lemma 8.3.

3. If $\partial D$ meets $\partial_{2} P, \gamma$ is parallel to $\partial_{1} P$, and the tunnel exits in $H_{1}^{R}$, then $\epsilon$ meets $b_{-2}^{R}, \ldots, b_{3}^{R}$ by Lemma 8.11. Note that $\alpha \subseteq E$ has endpoints in $b_{-2}^{R}$ and $d_{1}^{R}$. Furthermore, $\partial D$ meets $c^{R}$. Examining Figure 9 we see that $\epsilon$ cannot meet $b_{-1}^{R}$ by Lemma 8.3. Therefore $\epsilon$ meets $b_{-2}^{R}$ exactly twice.

Examining Figure 9 we see that Lemma 8.3 implies that any arc of $D \cap E$ with one endpoint in $c^{R}$ must have its other endpoint in either $b_{-2}^{R}$ or $b_{-2}^{P}$. The latter is not a possibility since $\partial D \cap P$ does not meet $b_{-2}^{P}$ in this case. In $D$, an arc with endpoint $c^{R}$ in a component of $\Delta$ has its other endpoint in $b_{-2}^{R}$ in $\epsilon$. Since $\epsilon$ meets $b_{-2}^{R}$ exactly twice and the other arc has endpoints $b_{-2}^{R}$ and $d_{1}^{R}$, there is exactly one component of $\Delta$. This component contains $p$ endpoints of arcs of intersection of $D \cap E$. However, $\epsilon$ contains at least $q+p-3$ endpoints of arcs of intersection of $D \cap E$ $(\epsilon \cap P$ contains $p-1$ endpoints of arcs, and $\epsilon \cap R$ contains at least $q-2)$. Since $q>3$, there is an arc violating Lemma 8.4.

4. If $\partial D$ meets $\partial_{1} P, \gamma$ is parallel to $\partial_{2} P$, and the tunnel exits in $H_{2}^{R}$, then $\gamma$ meets $b_{1}^{R}$. Recall that in this case $\beta$ has endpoints in $d_{-1}^{R}$ and $b_{2}^{R}$. Examining Figure 9 it is clear that there is an arc violating Lemma 8.3.

5. If $\partial D$ meets $\partial_{1} P, \gamma$ is parallel to $\partial_{2} P$, and the tunnel exits in $H_{1}^{R}$, then $\gamma$ meets both $c^{R}$ and $b_{-1}^{R}$. In this case $\beta$ has endpoints in $d_{1}^{R}$ and $b_{-1}^{R}$, and examining Figure 9 there must be an arc violating Lemma 8.3.

Therefore $\partial_{1} A$ is nontrivial in $\partial U$. Note that $\overline{U \backslash N(K)}$ is irreducible and not a product by Lemma 4.2. By [4, Lemma 2.5.3], $\partial_{2} A$ is either meridional on $\partial N(K)$ or meets a meridian of $\partial N(K)$ exactly once. Suppose that $\partial_{2} A$ is not meridional on $\partial N(K)$. By the above arguments, $A$ describes an isotopy of $K$ to $\partial U$ so that $K$ meets a component of $\partial Q$ zero or one times.

We want to examine this annulus in the context of the original construction of $K \subseteq H$ from section 3 . There, it is clear that after attaching a 2 -handle to $\partial_{1} P$ or $\partial_{2} P$ we obtain a knot which is isotopic to a curve in the boundary of the solid torus meeting the other components of $\partial P$ either $p$ or $q$ times. Thus there is an annulus $A^{\prime}$ properly embedded in $\overline{U \backslash N(K)}$ which meets a component of $\partial Q$ either $p$ or $q$ times. Since there are two annuli in the knot exterior with different slopes on $\partial N(K)$, the proof of [4, Lemma 2.5.3] shows that $\overline{U \backslash N(K)}$ must be a product. However, we have already seen that this is not the case.

Therefore $\partial_{2} A$ is meridional on $\partial N(K)$. We obtain a meridian disk for $U$ meeting $K$ once, which is impossible by Lemma 4.2 and the fact that $\gamma$ is not parallel to $\partial_{3} P$.

Now let $q=2 p \pm 1, p>1$, and $q>3$, and write $(M, H, K)=\left(M_{p, q}^{L}, H_{p, q}^{L}, K_{p, q}^{L}\right)$. As before, suppose that there is a tunnel $t$ as in Proposition 3.2 so that $M^{\prime}=$ $\overline{M \backslash(K \cup t)}$ is a handlebody, and suppose that $t$ lies entirely in $H$. 
Lemma 9.3. The surface $P \subseteq M^{\prime}$ is $\partial$-incompressible.

Proof. By Lemma 8.2, we need only examine $\partial$-compressing disks which meet $E$. Let $D$ be such a $\partial$-compressing disk for $P$ in $H^{\prime}$ which minimizes $(\mid D \cap$ $E||, D \cap \partial N(t)|,| \partial D \cap \partial E \cap P \mid)$. By Lemma 8.11 there is a component $\alpha$ of $D \cap E$, outermost in $D$, which meets either $d_{-1}$ and $b_{2}$ or $d_{1}$ and $b_{-2}$ in $R$.

Case 1: Suppose that $D$ is a $\partial$-compressing disk as above, $\partial D$ is nonseparating in $P$, and that $\alpha$ has endpoints in $d_{-1}$ and $b_{2}$. Recall that the proof of Lemma 8.11 shows that both components $\epsilon \cap R$ and $\epsilon \cap P$ are contained in the hexagonal regions of $R$ and $P$, respectively. Let $\delta$ be the component of $\Delta$ meeting $\beta$. Examining Figure 9 we see that $\partial D$ cannot meet $b_{1}^{R}$ without violating Lemma 8.3. Therefore $\delta$ is parallel to $\partial_{1} P$, and by Lemma 8.5 every component of $\Delta$ has label sequence $c, d_{1}, \ldots, d_{-1}$ or the reverse. Note that $\partial_{2} P$ and $\partial_{3} P$ lie in the same component of $R^{\prime} \backslash \Delta$. The arc $\epsilon$ meets $b_{2}$, and so by Lemma 9.2 and the fact that $\partial D$ is nonseparating, $\epsilon$ must meet $\partial_{1} P$. Recall from Remark 4.5 that $\partial_{2} P \cap a^{P}=\partial_{2} P \cap b_{1}^{R}$. A look at Figure 8 shows that on the side of $\beta, \epsilon$ must cross $b_{2}, b_{4}, \ldots b_{-1}$.

If $\epsilon$ does not meet any other arcs of $\partial E \cap R$, then by Lemma 8.4, Lemma 8.6, and Lemma 8.7, there is an outermost arc of $D$ connecting $b_{-1}$ to either $c$ or $d_{-1}$. The first arc is forbidden by Lemma 8.3. Therefore there is an arc in $E$ with endpoints in $b_{-1}$ and $d_{-1}$. Note that all arcs in $E$ connecting $b_{2}, b_{4}, \ldots b_{-3}$ to $c$ or any $d_{i}$ must cross this arc unless all the $b_{2 i}$ connect to $d_{-1}$. Since $q>4$, we may consider the subdisk of $D$ bounded by $\partial D, \alpha$, and the arc with endpoints $b_{4}$ and $d_{-1}$. If $p=2$, then $d_{1}^{R}=d_{-1}^{R}$. In light of Lemma 8.7, there must be an arc in this subdisk violating Lemma 8.3. If $p>2$, then by Lemma 8.7 the subdisk contains arcs violating Lemma 8.6.

Therefore $\epsilon$ must meet other arcs of $\partial E \cap R$. By Remark 4.5, it wraps around $\partial_{1} P$ at least once. The component of $\epsilon \backslash P$ meeting $d_{-1}$ has the same label set as the components of $\Delta$. We may treat this subarc of $\epsilon$ as another component of $\Delta$, and the arguments of Lemma 8.4, Lemma 8.6, and Lemma 8.7 give a contradiction.

Case 2: Suppose that $\partial D$ is nonseparating in $P$ and $\alpha$ has endpoints in $d_{1}$ and $b_{-2}$. Let $\delta$ be the component of $\Delta$ meeting $\beta$. By Lemma $8.5, \delta$ must meet $c^{R}$. Examining Figure 9 we see that it misses $b_{-1}$ by Lemma 8.3. Therefore $\delta$ is parallel to $\partial_{1} P$, and by Lemma 8.5 the label sequence of every component of $\Delta$ is $d_{1}, \ldots, d_{-1}, c$ or the reverse. Again, $\partial_{2} P$ and $\partial_{3} P$ lie in the same component of $R^{\prime} \backslash \Delta$. We see from Lemma 9.2 that $\epsilon$ must either meet $b_{-2}, b_{-4}, \ldots, b_{3}$ before passing to $P$, or it must meet $b_{-2}, b_{-4}, \ldots, b_{1}$ before passing to $P$.

Suppose that $\epsilon \cap R$ consists of exactly $b_{-2}, \ldots, b_{3}$. Then $d_{-1}^{R}=d_{1}^{R}$, and so $(p, q)=(2,5)$. In this case, $b_{3}$ is the only endpoint of a component of $D \cap E$ in $\epsilon$, and this violates Lemma 8.8.

If $p>2$, then $\epsilon$ ends after meeting either $c$ or $d_{1}$. The arc with this endpoint must be outermost by Lemma 8.6 and Lemma 8.7. Therefore there is an arc in 
$E$ with both endpoints on the same component of $\partial E \cap R$ or one with endpoints $c$ and $d_{1}$, both of which contradict Lemma 8.3.

So suppose that $\epsilon$ hits $b_{-2}, \ldots, b_{1}$ before traveling to $P$. As above, the last endpoint of $\epsilon$ must be either $c$ or $d_{1}$, and we get a contradiction with Lemma 8.3.

Case 3: If $\partial D$ separates $P$, note that the points $\epsilon \cap \partial R$ lie on the same component of $\partial R$. But this means that both components of $\epsilon \cap R^{\prime}$ lie in the same component of $R^{\prime} \backslash \Delta$, which violates Lemma 9.2.

\section{References}

[1] John Berge. Knots in handlebodies which can be surgered to produce handlebodies. Unpublished manuscript.

[2] John Berge. The knots in $D^{2} \times S^{1}$ which have nontrivial Dehn surgeries that yield $D^{2} \times S^{1}$. PhD thesis, University of Wisconsin, Madison, 1987 .

[3] John Berge. The knots in $D^{2} \times S^{1}$ which have nontrivial Dehn surgeries that yield $D^{2} \times S^{1}$. Topology and its Applications, 38(1):1 - 19, 1991 .

[4] M. Culler, C. McA. Gordon, J. Luecke, and P. B. Shalen. Dehn surgery on knots. Ann. Math., 125:237-300, 1987.

[5] Roberto Frigerio, Bruno Martelli, and Carlo Petronio. Dehn filling of cusped hyperbolic 3-manifolds with geodesic boundary. J. Differential Geom., 64(3):425-455, 2003.

[6] David Gabai. Surgery on knots in solid tori. Topology, 28(1):1-6, 1989.

[7] David Gabai. 1-Bridge braids in solid tori. Topology Appl., 37(3):221-235, 1990.

[8] C. McA. Gordon and J. Luecke. Knots are determined by their complements. Bull. Amer. Math. Soc. (N.S.), 20(1):83-87, 1989.

[9] William Jaco. Lectures on three-manifold topology. Published for the Conference Board of the Mathematical Sciences by the American Mathematical Society, Providence, R.I, 1980.

[10] Tsuyoshi Kobayashi. Classification of unknotting tunnels for two bridge knots. Geom. Topol. Monogr., 2:259-290, 1999.

[11] Yi Ni. Dehn surgeries on knots in product manifolds. Journal of Topology, 4(4):799-816, 2011.

[12] Richard S. Stevens. Classification of homotopy torus knot spaces. Proc. Amer. Math. Soc., 52:461-464, 1975.

[13] Ying Qing Wu. Immersed surfaces and Seifert fibered surgery on Montesinos knots. Trans. Amer. Math. Soc. To appear. 
[14] Ying Qing Wu. Incompressibility of surfaces in surgered 3-manifolds. Topology, 31(2):271-279, 1992.

[15] Ying Qing Wu. $\partial$-reducing Dehn surgeries and 1-bridge knots. Math. Ann., 295(2):319-331, 1993. 